\title{
High-order post-Newtonian contributions to the two-body gravitational interaction potential from analytical gravitational self-force calculations
}

\author{
Donato Bini ${ }^{1}$ and Thibault Damour ${ }^{2}$ \\ ${ }^{1}$ Istituto per le Applicazioni del Calcolo "M. Picone", CNR, I-00185 Rome, Italy and \\ ${ }^{2}$ Institut des Hautes Etudes Scientifiques, 91440 Bures-sur-Yvette, France
}

(Dated: July 26, 2018)

\begin{abstract}
We extend the analytical determination of the main radial potential describing (within the effective one-body formalism) the gravitational interaction of two bodies beyond the 4th post-Newtonian approximation recently obtained by us. This extension is done to linear order in the mass ratio by applying analytical gravitational self-force theory (for a particle in circular orbit around a Schwarzschild black hole) to Detweiler's gauge-invariant redshift variable. By using the version of black hole perturbation theory developed by Mano, Suzuki and Takasugi, we have pushed the analytical determination of the (linear in mass ratio) radial potential to the 6th post-Newtonian order (passing through 5 and 5.5 post-Newtonian terms). In principle, our analytical method can be extended to arbitrarily high post-Newtonian orders.
\end{abstract}

\section{INTRODUCTION AND SUMMARY OF NEW RESULTS}

This paper is a follow up of previous work [1], where we analytically determined the radial potential describing (within the effective one-body formalism (EOB) [25]) the gravitational interaction of two bodies (of masses $\left.\left(m_{1}, m_{2}\right)\right)$ at the 4 th post-Newtonian $(4 \mathrm{PN})$ approximation. At this order of approximation, this determination could be done to all orders in the symmetric mass ratio $\nu:=\mu / M=m_{1} m_{2} /\left(m_{1}+m_{2}\right)^{2}$. (Here $M:=m_{1}+m_{2}$ denotes the total mass and $\mu=m_{1} m_{2} /\left(m_{1}+m_{2}\right)$ the reduced mass of the system). More precisely, the $4 \mathrm{PN}$ contributions that are nonlinear in $\nu$ were derived from recent results of Jaranowski and Schäfer [6], while the contribution linear in $\nu$ was obtained by a combination of techniques. First, we used a recently discovered link 7-9] between the $O(\nu)$ piece of the EOB (gaugeinvariant) radial potential $A(u ; \nu)$ and the $O(\nu)$ piece of Detweiler's gauge-invariant "redshift" function $z_{1}(\Omega)$ [10], along a circular orbit of frequency $\Omega$. Second, we used a combination of gravitational self-force (GSF) techniques for analytically computing the $O(\nu)$ piece of $z_{1}(\Omega)$, namely: spherical-harmonics-mode-sum regularization [10, 11], and improved analytic black hole perturbation techniques developed by the Japanese relativity school 12 18.

In this paper we give details of our previous work [1], and present the extension of our GSF analysis to the 6th post-Newtonian (6PN) level (passing through 5 and 5.5 post-Newtonian levels). Our analysis will give us access to these high-PN order terms to linear order in $\nu$ only. However, we shall argue that the $5.5 \mathrm{PN}$ contribution is exactly linear in $\nu$. In addition, we suggest that the EOB formalism allows one to derive some of the coefficients of the higher powers of $\nu$ in the binding energy of a binary system.

We consider a two-body system with masses $m_{1}$ and $m_{2}$, moving on a circular orbit of (areal) radius $r_{0}$ and orbital frequency $\Omega$, in the limit where $m_{1} \ll m_{2}$. There are (at least) three ways to express our results. First, it can be expressed in terms of the version of Detweiler's gauge-invariant GSF function [10] that we shall actually compute, namely

$$
h_{k k}^{R}(u):=h_{\mu \nu}^{R} k^{\mu} k^{\nu} .
$$

Here $h_{\mu \nu}$ denotes the (mass-ratio rescaled) 1GSF metric perturbation of a Schwarzschild metric of mass $m_{2}: \quad \delta g_{\mu \nu}=g_{\mu \nu}\left(x ; m_{1}, m_{2}\right)-g_{\mu \nu}^{\text {Schwarzschild }}\left(x ; m_{2}\right)=$ $\left(m_{1} / m_{2}\right) h_{\mu \nu}+O\left(m_{1}^{2} / m_{2}^{2}\right) ; k^{\mu}$ denotes the helical Killing vector $k^{\mu} \partial_{\mu}=\partial_{t}+\Omega \partial_{\varphi}$ of the perturbed metric; and the superscript $R$ denotes the regularized value of $h_{\mu \nu}$ on the word line of the small mass $m_{1}$. In addition, the argument $u$ in the function $h_{k k}^{R}(u)$ denotes here $G M / c^{2} r_{0}$, where $r_{0}$ is the radius of the considered circular orbit. Equivalently, one could consider that $u=\left(G M \Omega / c^{3}\right)^{2 / 3}$. Indeed, in a first-order GSF function, such as $h_{k k}^{R}(u)$, the argument need only be defined with background accuracy, i.e. modulo corrections of order $m_{1} / m_{2}$. In the following, the independent variable $u$ will be treated as a mathematical argument (varying between 0 and $+\infty$ ) whose physical meaning depends on the considered dependent variable.

We shall analytically compute the coefficients entering the $6 \mathrm{PN}$-accurate expansion (in powers of $u$ ) of $h_{k k}^{R}(u)$, i.e. the coefficients $h_{n}$ in

$$
\begin{aligned}
& h_{k k}^{R}(u)=h_{1} u+h_{2} u^{2}+h_{3} u^{3}+h_{4} u^{4}+h_{5}(\ln u) u^{5} \\
& \quad+h_{6}(\ln u) u^{6}+h_{6.5} u^{13 / 2}+h_{7}(\ln u) u^{7}+o\left(u^{7}\right) .
\end{aligned}
$$

Note that a term of order $u^{n}$ in this expansion corresponds to the $(n-1)-\mathrm{PN}$ level. The new terms that we shall analytically derive below are: (i) the nonlogarithmic part of the $5 \mathrm{PN}$ coefficient $h_{6}(\ln u)$ (its logarithmic part had been derived in [19]), (ii) the $5.5 \mathrm{PN}$ coefficient $h_{6.5}$, and (iii) the $6 \mathrm{PN}$ coefficient $h_{7}(\ln u)$ (which includes a logarithm of $u$ ). While we were ready to submit the present manuscript, a preprint by Shah et al. [20] appeared on the archives, and reported on an independent analytical derivation of the value of $h_{6.5}$, as well as on a plausible numerical-analytical derivation of the logarithmic term in $h_{7}$. See below for a detailed comparison of our results to the (mainly numerical) results of [20]. 
A second way to phrase our results is in terms of the first-order GSF contribution to the (gauge-invariant) EOB radial interaction potential $A\left(r ; m_{1}, m_{2}\right)=A(u ; \nu)$ of a general relativistic two-body system. Here, the argument $u$ denotes $u:=G M / c^{2} r$, with $r$ denoting the (gauge-invariant) EOB radial coordinate. The GSF expansion of the radial potential $A(u ; \nu)$ reads

$$
A(u ; \nu)=1-2 u+\nu a(u)+O\left(\nu^{2}\right) .
$$

Here the $\nu \rightarrow 0$ limiting value $1-2 u$ corresponds to the well-known Schwarzschild potential $A^{S}(u)=1-$ $2 G M / c^{2} r=1-2 u$, while $a(u)$ represents the first-order GSF (1GSF) modification of the radial potential $A(u ; \nu)$.

In this work we shall use the simple link found in Refs. 7] 9], namely

$$
a(u)=-\frac{1}{2} h_{k k}^{R}-\frac{u(1-4 u)}{\sqrt{1-3 u}},
$$

to deduce from our results on $h_{k k}^{R}(u)$ the PN expansion of the $1 \mathrm{GSF}$ coefficient $a(u)$ up to the $6 \mathrm{PN}$ level, i.e.

$$
\begin{aligned}
a(u) & =a_{3} u^{3}+a_{4} u^{4}+a_{5}(\ln u) u^{5}+a_{6}(\ln u) u^{6} \\
& +a_{6.5} u^{13 / 2}+a_{7}(\ln u) u^{7}+o\left(u^{7}\right) .
\end{aligned}
$$

As in the case of the $u$ expansion of $h_{k k}^{R}(u)$ above, a term of order $u^{n}$ in this expansion corresponds to the $(n-1)$ PN level. The new terms in the expansion (5) we shall analytically determine are: (i) the non-logarithmic part of the $5 \mathrm{PN}$ coefficient $a_{6}(\ln u)$ (its logarithmic part had been derived in 7, 21] ), (ii) the $5.5 \mathrm{PN}$ coefficient $a_{6.5}$, and (iii) the $6 \mathrm{PN}$ coefficient $a_{7}(\ln u$ ) (which includes a logarithm of $u$ ).

A third way to express our results is in terms of the (gauge-invariant) function relating the binding energy $E_{B}=H^{\text {tot }}-M c^{2}$ of a circular orbit to its orbital frequency $\Omega$. Using the convenient dimensionless frequency parameter $x:=\left(G M \Omega / c^{3}\right)^{2 / 3}$, it has the form

$$
\begin{aligned}
E_{B}(x ; \nu)= & -\frac{1}{2} \mu c^{2} x\left(1+e_{1 \mathrm{PN}}(\nu) x+e_{2 \mathrm{PN}}(\nu) x^{2}\right. \\
& +e_{3 \mathrm{PN}}(\nu) x^{3}+e_{4 \mathrm{PN}}(\nu, \ln x) x^{4} \\
& +e_{5 \mathrm{PN}}(\nu, \ln x) x^{5}+e_{5.5 \mathrm{PN}}(\nu) x^{5.5} \\
& \left.+e_{6 \mathrm{PN}}(\nu, \ln x) x^{6}+o\left(x^{6}\right)\right) .
\end{aligned}
$$

Below, we shall deduce from our results on the EOB radial potential (using simple EOB-derived results [3, 22]) the analytical expressions of the $1 \mathrm{GSF}$ ( $\nu$-linear) pieces of $e_{5 \mathrm{PN}}, e_{5.5 \mathrm{PN}}$, and $e_{6 \mathrm{PN}}$. We shall also indicate several $\nu$ nonlinear terms in the $\nu$-dependent coefficients $e_{\mathrm{nPN}}(\nu)$, which arguably follow from the special structure of the EOB formalism (within which the $\nu$ dependence of the radial potential $A(u, \nu)$ incorporates remarkable cancellations, not present in $\left.E_{B}(x ; \nu)\right)$.

The presence of (conservative) contributions at the 5.5 PN level (i.e. $\left.O\left(1 / c^{11}\right)\right)$ in the various functions mentioned above seems to conflict with the usual PN lore that conservative (time-even) effects arise at even powers of $1 / c$, and that odd powers of $1 / c$ are associated with time-odd radiation-reaction effects . As we shall discuss in detail in Sec. IIIB below, this unexpected result can be heuristically understood as stemming from some results of Blanchet and Damour [23, 24] about hereditary effects (given by an integral over the past behavior of the source) in the inner metric of the system, and their relation (via energy balance) to corresponding hereditary effects ("radiative tail") in the wave-zone metric. We shall find that our analytical results for the 5.5PN-level (i.e. $O\left(1 / c^{11}\right)$ ) quantities $h_{6.5}$ and $a_{6.5}$ arise from the inner-metric image of the second-order radiative tail 25]. The important conceptual point here is (as already emphasized in 23]) that the hereditary tail effects are time dissymetric without being time anti-symmetric. They can thereby generate terms that are either radiation-reaction-like or conservative (thereby violating the usual PN lore). [After the submission of the present work, there appeared on the archives a paper by Blanchet et al. [26] which confirmed our (heuristic) analysis by a detailed multipolar-postMinkowskian treatment of the gravitational field outside the source.]

\section{ANALYTICAL COMPUTATION OF CONSERVATIVE GSF EFFECTS ALONG CIRCULAR ORBITS}

\section{A. General strategy for computing $h_{k k}^{R}$}

Detweiler 10] has pointed out the existence of one simple, (conservative) gauge-invariant function, available within first-order $(O(\nu))$ GSF theory, associated with the sequence of circular orbits of an extreme mass-ratio binary system: $m_{1} \ll m_{2}$. Computing the $O(\nu)$ piece of this redshift function $z_{1}(\Omega ; \nu) \equiv 1 / u_{1}^{t}(\Omega ; \nu)$ is equivalent [10, 27, 28] to computing the regularized value, along the world line $y_{1}^{\mu}$ of the small mass $m_{1}$, of the double contraction of the $O\left(m_{1} / m_{2}\right)$ metric perturbation $h_{\mu \nu}$ (considered in an asymptotically flat gauge)

$g_{\mu \nu}\left(x ; m_{1}, m_{2}\right)=g_{\mu \nu}^{(0)}\left(x ; m_{2}\right)+\frac{m_{1}}{m_{2}} h_{\mu \nu}(x)+O\left(\left(\frac{m_{1}}{m_{2}}\right)^{2}\right)$

(where $g_{\mu \nu}^{(0)}\left(x ; m_{2}\right)=g_{\mu \nu}\left(x ; m_{1}=0, m_{2}\right)$ is a Schwarzschild metric of mass $\left.m_{2}\right)$, with the four-velocity $u_{1}^{\mu}=d y_{1}^{\mu} / d s_{1}$ of $m_{1}$, say

$$
h_{u u}^{R}:=\operatorname{Reg}_{x \rightarrow y_{1}}\left[h_{\mu \nu}(x) u_{1}^{\mu} u_{1}^{\nu}\right] .
$$

Following Refs. 10, 11, 17, 27] the regularization operation indicated in Eq. (8) is done by subtracting the (leading-order) singular part in the spherical harmonics expansion of $h_{u u}$. This yields $h_{u u}^{R}$ as a series indexed by $l=0,1,2, \ldots$

$$
h_{u u}^{R}=\sum_{l=0}^{\infty}\left(h_{u u}^{(l)}-D_{0}\right)
$$


where

$$
h_{u u}^{(l)}=\sum_{m=-l}^{+l} u^{\mu} u^{\nu} h_{\mu \nu}^{(l, m)}
$$

and where the (l-independent) subtraction constant $D_{0}$ is known [10, 29] to be

$$
\begin{aligned}
D_{0} & =2 u \sqrt{\frac{1-3 u}{1-2 u}} F\left(\frac{1}{2}, \frac{1}{2}, 1 ; \frac{u}{1-2 u}\right) \\
& =2 u \sqrt{\frac{1-3 u}{1-2 u}}\left(\frac{2}{\pi} K(k)\right) .
\end{aligned}
$$

Here, $u=G m_{2} / c^{2} r_{0}=G M / c^{2} r_{0}+O(\nu), F(a, b, c ; z)$ is Gauss's hypergeometric function and $K(k)=$ $\int_{0}^{\pi / 2} d \theta / \sqrt{1-k^{2} \sin ^{2} \theta}$ is the complete elliptic integral of the first kind with modulus $k=\sqrt{u /(1-2 u)}$. The series (9) of "subtracted" l-modes, $h_{u u}^{(l) \text { subtracted }}:=h_{u u}^{(l)}-D_{0}$, converges like $\sum_{l} 1 / l^{2}$.

As already mentioned, we find more convenient to work with the double contraction $h_{k k}:=h_{\mu \nu} k^{\mu} k^{\nu}$ with the helical Killing vector $k^{\mu} \partial_{\mu}=\partial_{t}+\Omega \partial_{\varphi}$ (such that $k^{\mu}=$ $\left(d s_{1} / d t\right) u_{1}^{\mu}$ with $\left.d s_{1} / d t=\sqrt{1-3 u}\right)$, i.e.

$$
h_{k k}^{R}=(1-3 u) h_{u u}^{R}=\sum_{l}\left(\left(\sum_{m} h_{k k}^{(l, m)}\right)-\tilde{D}_{0}\right)
$$

with a renormalized subtraction constant $\tilde{D}_{0}:=(1-$ $3 u) D_{0}$.

Let us note for completeness the relation between $h_{k k}^{R}(u)$ and other versions of Detweiler's redshift variable, such as

$$
h_{u u}^{R}:=h_{\mu \nu}^{R} u_{1}^{\mu} u_{1}^{\nu}=\frac{1}{1-3 u} h_{k k}^{R}
$$

or

$$
u_{(1 G S F)}^{t}=\frac{1}{2} \frac{1}{\sqrt{1-3 u}} h_{u u}^{R}=\frac{1}{2} \frac{1}{(1-3 u)^{3 / 2}} h_{k k}^{R} .
$$

The argument $u$ in $h_{k k}^{R}(u)$ or $1-3 u$ should not be confused with the notation $u_{1}^{\mu}$ used for the 4-velocity of the small particle labelled 1. [In turn the particle label 1 should not be confused with the use of 1 as a label for 1GSF contributions. To avoid such a confusion we have suppressed the particle label on $u^{t}$ in the last equation.]

In previous works [10, 20, 27, 28, 30], $h_{u u}^{R}$ was evaluated numerically along a (discrete) sequence of circular orbits [parametrized by $r, u$ or $x=\left(G M \Omega / c^{3}\right)^{2 / 3}=u+O(\nu)$ ].

On the analytical side, $h_{u u}^{R}$ (or equivalent functions) was evaluated by means of usual PN theory at increasing levels of accuracy: 2PN [10], and 3PN [27]. The logarithmic contributions to $h_{u u}^{R}$ were evaluated at 4PN in [19, 22], and even at $5 \mathrm{PN}$ in [19]. In our previous work [1] we showed how to analytically compute the PN expansion of $h_{k k}^{R}=(1-3 u) h_{u u}^{R}$ by using black hole perturbation theory. Such an approach avoids the subtleties linked to the well-known breakdown of the usual postNewtonian expansion arising at the 4PN level [23]. More precisely, our approach is based on computing the postMinkowskian expansion of the function $h_{k k}^{R}(u)$ (weakfield expansion in powers of $G$, or equivalently in powers of $u=G M / c^{2} r$ ), by using the version of Regge-WheelerZerilli (RWZ) black hole perturbation theory that has been developed by Mano, Suzuki and Takasugi (MST) 12 14. [Let us note that Refs. 15 18 made valient attempts to apply the MST formalism to GSF theory, but were bogged down by gauge-dependent issues. The fact that we consider a gauge-invariant quantity allows us to get novel results.] In our short report [1], we only gave the result of our analysis at the $4 \mathrm{PN}$ level, by providing the analytical expression of the non-logarithmic coefficient entering $h_{k k}^{R}(u)$ at $4 \mathrm{PN}$ (i.e. the coefficient of $u^{5}$ ). In the present paper, we report on our higher-PN results: at $5 \mathrm{PN}, 5.5 \mathrm{PN}$ and $6 \mathrm{PN}$.

\section{B. Solving in terms of their sources the radial functions $R_{l m \omega}^{\text {(odd) }}, R_{l m \omega}^{(\text {even })}$ and $Z_{l m \omega}(r)$ entering the Regge-Wheeler-Zerilli metric perturbation}

In the series (12) over $l$, the low-multipole contributions $l=0$ and $l=1$ (even and odd) can be computed from the corresponding exact results of Zerilli [31]. Note the subtlety that we are computing here $h_{k k}^{R}(u)$ in an asymptotically flat gauge. One must accordingly rephrase the classic results of Zerilli 31]. We recall in this respect that the $l=0$ contribution to the Lorenz-gauge metric perturbation fails to be (manifestly) asymptotically flat 32, 33. When using the Lorenz gauge, one must accordingly introduce a correction to compute flat-gauge quantities [22, 28, 30].

On the other hand, the "dynamical" multipoles of order $l \geq 2$ are more difficult to evaluate. We started from the corrected version of the RWZ equations derived by Sago, Nakano and Sasaki [15]. [Beware, however, that the sign of the source term $B_{l m}^{(0)}$ in Table I there should be $-i(\ldots)$ instead of $+i(\ldots)$; the later papers [16, 17] give the correct sign.]

The original RWZ formalism expresses an odd-parity metric perturbation $h_{\mu \nu}^{(l, m)}$, with frequency $\omega$, in terms of a radial function $R_{l m \omega}^{\text {(odd) }}(r)$ satisfying a ReggeWheeler(RW)-type equation

$$
\mathcal{L}_{(\mathrm{RW})}^{(r)}\left[R_{l m \omega}^{(\mathrm{odd})}\right]=S_{l m \omega}^{(\mathrm{odd})}(r) .
$$

Here $\mathcal{L}_{(\mathrm{RW})}^{(r)}$ denotes the RW operator

$$
\begin{aligned}
\mathcal{L}_{(\mathrm{RW})}^{(r)} & =\left(1-\frac{2 M}{r}\right)^{2} \frac{d^{2}}{d r^{2}}+\frac{2 M}{r^{2}}\left(1-\frac{2 M}{r}\right) \frac{d}{d r} \\
& +\left[\omega^{2}-V_{(\mathrm{RW})}(r)\right] \\
& =\frac{d^{2}}{d r_{*}^{2}}+\left[\omega^{2}-V_{(\mathrm{RW})}(r)\right]
\end{aligned}
$$


with $d / d r_{*}=f(r) d / d r($ with $f(r):=1-2 M / r)$, and a RW potential

$$
V_{(\mathrm{RW})}(r)=\left(1-\frac{2 M}{r}\right)\left(\frac{l(l+1)}{r^{2}}-\frac{6 M}{r^{3}}\right) .
$$

Note that we denote here the large background mass (which, strictly speaking, should be $m_{2}$ ) by $M$. This is allowed in our calculation which is to linear order in $m_{1} / m_{2}$. (Here, as often in the following, we omit to include a label $l \omega$ indicating the $l \omega$-dependence of various objects.) The odd-parity source term in Eq. (15) (given by Eq. (A35) of [15]) is of the form

$$
S_{l m \omega}^{(\mathrm{odd})}(r)=s_{0}^{(\mathrm{o})} \delta\left(r-r_{0}\right)+s_{1}^{(\mathrm{o})} \delta^{\prime}\left(r-r_{0}\right),
$$

where $r_{0}$ denotes the radius of the circular orbit of particle 1.

On the other hand, the original RWZ formalism expresses a (monochromatic) even-parity $h_{\mu \nu}^{(l, m)}$ in terms of a radial function $Z_{l m \omega}(r)$ satisfying a Zerilli-type equation 31], involving a more complicated potential than the RW equation (15). Using a result of Chandrasekhar 34], one can, however, replace the pair $\mathcal{Z}_{l m \omega}:=\left(Z_{l m \omega}(r), d Z_{l m \omega}(r) / d r\right)$ by a new pair of functions, say $\mathcal{R}_{l m \omega}:=\left(R_{l m \omega}^{(\text {even })}(r), d R_{l m \omega}^{(\text {even })}(r) / d r\right)$, satisfying a simpler RW-type equation, say

$$
\mathcal{L}_{(\mathrm{RW})}^{(r)}\left[R_{l m \omega}^{(\text {even })}\right]=S_{l m \omega}^{(\text {even })}(r) .
$$

The price to pay for this simplification of the potential is: (i) the transformation between $\mathcal{Z}_{l m \omega}$ and $\mathcal{R}_{l m \omega}$ involves source terms, and (ii) the new even-parity source term is connected to the original Zerilli one by an expression of the form $S_{l m \omega}^{(\mathrm{even})}=\mathcal{A}_{11}(r) S_{l m \omega}^{(\mathrm{Z})}+\mathcal{A}_{12}(r) \frac{d}{d r}\left(S_{l m \omega}^{(\mathrm{Z})}\right)$. As a consequence the new even-parity source term in Eq. (19) is of the form

$$
S_{l m \omega}^{(\text {even })}=s_{0}^{(\mathrm{e})} \delta\left(r-r_{0}\right)+s_{1}^{(\mathrm{e})} \delta^{\prime}\left(r-r_{0}\right)+s_{2}^{(\mathrm{e})} \delta^{\prime \prime}\left(r-r_{0}\right) .
$$

At this stage, the problem is reduced to solving some RW equation (one for each $l m \omega$ and each parity) with given (distributional) source terms. The source terms derive from the spherical harmonics projection of the distributional stress-energy tensor of particle 1:

$$
T_{1}^{\mu \nu}\left(x^{\lambda}\right)=m_{1}(-g)^{-1 / 2} \int d s_{1} u_{1}^{\mu} u_{1}^{\nu} \delta^{(4)}\left(x^{\lambda}-y_{1}^{\lambda}\left(s_{1}\right)\right) .
$$

As a consequence, in the case of a circular orbit, the (discrete) frequencies entering $T_{1}^{\mu \nu}(x)$ (and therefore $h_{\mu \nu}(x)$ ) are related to the basic orbital frequency $\Omega=d \phi_{1} / d t_{1}$ and the "magnetic" number $m$ by $\omega=\omega_{m}:=m \Omega$.

The solution of the RW equations (15), 19) is determined by the choice of the boundary conditions incorporated in a Green's function $G\left(r, r^{\prime}\right)$ normalized so as to satisfy

$$
\mathcal{L}_{(\mathrm{RW})}^{(r)} G\left(r, r^{\prime}\right)=f\left(r^{\prime}\right) \delta\left(r-r^{\prime}\right)
$$

Such a Green's function can be generally expressed in terms of two, specially chosen, independent homogeneous solutions of the RW operator (and of the Heaviside step function $H(x)$ )

$$
\begin{aligned}
G\left(r, r^{\prime}\right) & =\frac{1}{W}\left[X_{(\mathrm{in})}(r) X_{(\mathrm{up})}\left(r^{\prime}\right) H\left(r^{\prime}-r\right)\right. \\
& \left.+X_{(\mathrm{in})}\left(r^{\prime}\right) X_{(\mathrm{up})}(r) H\left(r-r^{\prime}\right)\right] \\
& \equiv G_{(\mathrm{in})}\left(r, r^{\prime}\right) H\left(r^{\prime}-r\right)+G_{(\mathrm{up})}\left(r, r^{\prime}\right) H\left(r-r^{\prime}\right),
\end{aligned}
$$

where

$$
\mathcal{L}_{(\mathrm{RW})}^{(r)} X_{(\mathrm{in})}(r)=0=\mathcal{L}_{(\mathrm{RW})}^{(r)} X_{(\mathrm{up})}(r),
$$

and where $W$ denotes the Wronskian

$$
\begin{aligned}
W & =\left(1-\frac{2 M}{r}\right)\left[X_{(\text {in })}(r) \frac{d}{d r} X_{(\text {up })}(r)\right. \\
& \left.-\frac{d}{d r} X_{(\text {in })}(r) X_{(\text {up })}(r)\right]=\text { constant } .
\end{aligned}
$$

The physical Green's function we are interested in is the retarded one. It is obtained, as usual, by choosing for $X_{(\mathrm{in})}^{l \omega}$ a solution that is incoming from $r=+\infty$ (and purely ingoing on the horizon), and for $X_{(\mathrm{up})}^{l \omega}$ a solution that is upgoing from the horizon (and purely outgoing at infinity). This uniquely determines the solutions of the inhomogeneous even-parity and odd-parity RW equations, namely

$$
R_{l m \omega}^{(\text {even/odd })}(r)=\int d r^{\prime} G\left(r, r^{\prime}\right) f\left(r^{\prime}\right)^{-1} S_{l m \omega}^{(\text {even/odd })}\left(r^{\prime}\right) .
$$

Note that the distributional nature of the radial source functions, notably $S_{l m \omega}^{\text {even }}(r) \ni \delta^{\prime \prime}\left(r-r_{0}\right)$, implies that, e.g., $R_{l m \omega}^{\text {even }}(r)$ is not only discontinuous as $r$ crosses $r_{0}$, but (formally) contains a contribution $\propto \delta\left(r-r_{0}\right)$.

\section{Evaluating the even and odd multipolar pieces $h_{k k, l m}^{\text {(even/odd) }}$ of $h_{k k}$ in terms of homogeneous solutions} $X_{l \omega}^{(\mathrm{in})}, X_{l \omega}^{(\mathrm{up})}$ of the Regge-Wheeler equation

Having determined $R_{l m \omega}^{\text {(even/odd) }}(r)$ by the (distributional) formula (26), one can then compute the original Zerilli radial functions $\left(Z_{l m \omega}(r), d Z_{l m \omega}(r) / d r\right)$, and thereby evaluate the metric perturbation $h_{\mu \nu}^{(l m \omega)}(r)$, and, in particular, the double contraction

$$
\begin{aligned}
h_{k k}(t, r, \theta, \phi) & =h_{t t}^{(\mathrm{RWZ})}+2 \Omega h_{t \phi}^{(\mathrm{RWZ})}+\Omega^{2} h_{\phi \phi}^{(\mathrm{RWZ})} \\
& =\sum_{l, m} e^{-i \omega_{m} t} h_{k k_{l m \omega}}^{(\mathrm{RWZ})}
\end{aligned}
$$

we are interested in. Here, $h_{k k}\left(x^{\lambda}\right)$ is evaluated at a generic field point $x^{\lambda}=(t, r, \theta, \phi)$. The next step is to restrict $t, \theta$ and $\phi$ to the values corresponding to the considered instantaneous position of particle 1, say 
$t, \theta=\pi / 2$ and $\phi=\phi_{1}(t)=\Omega t$ (in the equatorial plane of the background Schwarzschild metric). [This eliminates the apparent $t$-dependence of $h_{k k}$ in Eq. (27).] At this stage, $h_{k k}$ depends only on $r$. Considering the two limits $r \rightarrow r_{0}^{-}$and $r \rightarrow r_{0}^{+}$, we have checked that they yield the same result for the value of $h_{k k}$ at the location $r=r_{0}$ of particle 1. [This confirms the idea of Detweiler 10] that the gauge-invariant quantity $h_{u u}^{R}$ can be correctly evaluated on the world line of $y_{1}$ even if one uses a gauge (such as the RWZ one) where $h_{\mu \nu}\left(x ; y_{1}\right)$ has a worse behaviour than its Lorenz-gauge version.]

Our final result for $h_{k k}^{(l, m)} \equiv h_{k k}^{(l, m)}\left(r_{0}\right)$, which enters Eq. (12), is the sum of an even and an odd contribution

$$
h_{k k}^{(l, m)}=h_{k k, l m}^{(\text {even })}+h_{k k, l m}^{(\text {odd })}
$$

The odd contribution takes the rather simple form

$$
\begin{aligned}
h_{k k, l m}^{(\text {odd })}= & -\left|\partial_{\theta} Y_{l m}(\pi / 2,0)\right|^{2} \frac{8 \pi \Gamma}{r_{0}^{3} W \Lambda} M f_{0}^{2} \times \\
& \times\left[r_{0} \frac{d X_{l \omega}^{(\text {in })}}{d r_{0}}+X_{l \omega}^{(\text {in })}\right]\left[r_{0} \frac{d X_{l \omega}^{(\text {up })}}{d r_{0}}+X_{l \omega}^{(\text {up })}\right] .
\end{aligned}
$$

Here $\Gamma:=u_{1}^{t}=(1-3 u)^{-1 / 2}$, and $\Lambda:=\frac{1}{4}(l-1) l(l+1)(l+$ $2)$.

The corresponding result for $h_{k k, l m}^{(\text {even })}$ is more complicated, and reads

$$
\begin{aligned}
h_{k k, l m}^{(\text {even })}= & -\left|Y_{l m}(\pi / 2,0)\right|^{2} \frac{8 \pi \Gamma}{r_{0}^{5} \Lambda W\left(r_{0}^{3} \Lambda^{2}+9 M^{3} m^{2}\right)} \times \\
& \times \mathcal{F}\left(X^{(\text {in })} ; r_{0}\right) \mathcal{F}\left(X^{(\text {up })} ; r_{0}\right)
\end{aligned}
$$

where

$$
\mathcal{F}\left(X ; r_{0}\right)=\tilde{\alpha}\left(r_{0}\right) \frac{d X\left(r_{0}\right)}{d r_{0}}+\tilde{\beta}\left(r_{0}\right) X\left(r_{0}\right)
$$

with (using the notation $\lambda:=(l-1)(l+2) / 2$ such that $\Lambda=\lambda(\lambda+1))$

$$
\begin{aligned}
\tilde{\alpha}\left(r_{0}\right)= & r_{0}^{2} f_{0}\left[\Lambda r_{0}^{2} f_{0}+3 M^{2}\left(\lambda+1-m^{2}\right)\right] \\
\tilde{\beta}\left(r_{0}\right)= & r_{0}^{2}\left(r_{0}-M\right) \lambda^{3}+r_{0}\left[6 M^{2}-r_{0}\left(5 M-2 r_{0}\right.\right. \\
& \left.\left.+M m^{2}\right)\right] \lambda^{2} \\
+ & \left(r_{0}^{3}-4 M r_{0}^{2}-M r_{0}^{2} m^{2}+9 M^{2} r_{0}-6 M^{3}\right) \lambda \\
& -3 M r_{0} f_{0}\left(m^{2}-1\right) .
\end{aligned}
$$

Note that both type of expressions have the usual "one-loop" structure: (source) $)^{*} \times($ propagator $) \times($ source $)$ (where the star indicates taking a complex conjugate, as appropriate for a transition amplitude). For the even case, the source term is proportional to $Y_{l m}(\pi / 2,0)$, while, for the odd case, the source term is proportional to $\partial_{\theta} Y_{l m}(\pi / 2,0)$. In both cases the propagator has the characteristic Green's function structure, i.e. it is bilinear in the homogeneous RW solutions $X_{l \omega}^{(\mathrm{in})}$ and $X_{l \omega}^{(\mathrm{up})}$, or their derivatives.

\section{Strategy for analytically computing the PN expansion of $h_{k k}^{R}(u)$}

To evaluate $h_{k k}^{R}$ from the RWZ results (29), (30) one still needs, according to Eq. (12), to: (i) sum over $m$ from $-l$ to $+l$; (ii) subtract $\tilde{D}_{0}$; and, finally, (iii) sum the result of (i) and (ii) over $\ell=0,1,2, \ldots$ Even the first (finite) sum over $m$ is quite nontrivial to compute analytically because one must remember that the index $\omega$ on the two solutions $X_{l \omega}^{(\mathrm{in})}, X_{l \omega}^{(\mathrm{up})}$ entering (29), (30) actually refers to $\omega_{m}=m \Omega$, not to mention the fact that one needs to obtain explicit, analytic expressions for the two homogeneous solutions $X_{l \omega}^{(\text {in) }}(r)$ and $X_{l \omega}^{(\text {up })}(r)$. The latter problem has been formally solved by Mano et al. 12 14] who gave analytic expressions for $X_{l \omega}^{(\text {in) }}$ and $X_{l \omega}^{(\text {up })}$ in the form of series of hypergeometric functions (of the usual, Gauss, type for $X^{(\text {in) }}$ and of the confluent type for $\left.X^{(\text {up) }}\right)$. More precisely, they obtained expressions of the type

$$
\begin{aligned}
X_{l \omega}^{(\mathrm{in})}(r)= & C_{(\mathrm{in})}^{\nu}(x) \sum_{n=-\infty}^{+\infty} a_{n}^{\nu} \times \\
& \times \bar{F}(n+\nu-1-i \epsilon,-n-\nu-2-i \epsilon, 1-2 i \epsilon ; x]
\end{aligned}
$$

$$
\begin{aligned}
X_{l \omega}^{(\mathrm{up})}(r)= & C_{(\mathrm{up})}^{\nu}(z) \sum_{n=-\infty}^{+\infty} a_{n}^{\nu}(-2 i z)^{n} \times \\
& \times \bar{\Psi}(n+\nu+1-i \epsilon, 2 n+2 \nu+2 ;-2 i z) .
\end{aligned}
$$

Here, $x=1-c^{2} r / 2 G M, z=\omega r / c, \epsilon=2 G M \omega / c^{3}=$ $2 m G M \Omega / c^{3}$

$$
\begin{aligned}
\nu= & l+\frac{1}{2 l+1}\left[-2-\frac{s^{2}}{l(l+1)}\right. \\
& +\frac{\left[(l+1)^{2}-s^{2}\right]^{2}}{(2 l+1)(2 l+2)(2 l+3)} \\
& \left.-\frac{\left(l^{2}-s^{2}\right)^{2}}{(2 l-1) 2 l(2 l+1)}\right] \epsilon^{2}+O\left(\epsilon^{4}\right),
\end{aligned}
$$

is an $\epsilon$-modified avatar of $l\left[12-14\right.$ (with $s^{2}=4$ in the present spin 2 case; see appendix A for more details), and

$$
\begin{gathered}
C_{(\mathrm{in})}^{\nu}(x)=c_{(\mathrm{in})} e^{i \epsilon[(x-1)-\ln (-x)]}(1-x)^{-1}, \\
C_{(\mathrm{up})}^{\nu}(z)=c_{(\mathrm{up})} e^{i z} z^{\nu+1}\left(1-\frac{\epsilon}{z}\right)^{-i \epsilon} 2^{\nu} e^{-\pi \epsilon} e^{-i \pi(\nu+1)}, \\
\bar{F}(a, b, c ; x)=\frac{\Gamma(a) \Gamma(b)}{\Gamma(c)} \quad F(a, b, c ; x), \\
\bar{\Psi}(a, b ; \zeta)=\frac{\Gamma(a-2) \Gamma(a)}{\Gamma\left(a^{*}\right) \Gamma\left(a^{*}+2\right)} \Psi(a, b ; \zeta)
\end{gathered}
$$

(with $a^{*}$ denoting the complex conjugate of $a$; and $\Psi$ the second Kummer function). Here the quantities $c_{(\text {in })}=$ 
$\eta^{\alpha_{(\text {in })}^{(l)}}$ and $c_{(\text {up })}=\eta^{\alpha_{(\text {up })}^{(l)}}$ are some $l$-dependent powers of $\eta:=1 / c$ that are defined so that $C_{(\text {in) }}^{\nu}(x)$ and $C_{(\text {up })}^{\nu}(z)$ both start with zeroth order in $\eta$. For example, for $l=2$ we take $c_{(\text {in })}=\eta^{-2}$ and $c_{(\text {up })}=\eta^{-3}$ so that

$$
\begin{aligned}
& C_{(\text {in })}(r)=\frac{2 M}{r}-2 i M \omega \eta-M r \omega^{2} \eta^{2}+O\left(\eta^{3}\right) \\
& C_{(\text {up })}(r)=-4 \omega^{3} r^{3}-4 i \omega^{4} r^{4} \eta+2 \omega^{5} r^{5} \eta^{2}+O\left(\eta^{3}\right) .
\end{aligned}
$$

Finally, the two-sided sequence of coefficients $a_{n}^{\nu}$ entering both series (33) and (34) are obtained by solving a three-term recursion relation, $\alpha_{n}^{\nu} a_{n+1}^{\nu}+\beta_{n}^{\nu} a_{n}^{\nu}+\gamma_{n}^{\nu} a_{n-1}^{\nu}=$ 0 , obtained by Mano et al. (see Eqs. (2.5)-(2.8) in [13]). We have solved this three-term relation between $n=-11$ and $n=+13$ (included) by initiating the recursion with $a_{13}=0$ and $a_{-11}=0$. This is sufficient for getting the crucial hypergeometric-expanded $X_{l \omega}^{(\mathrm{up})}$ to the $6 \mathrm{PN}$ level (see below).

In principle, the expressions (33), (34) can be used to compute $h_{k k}^{(l, m)}\left(r_{0}\right)$ for all values of $l$ and $m$. However, it would be difficult to use them to treat analytically the case of arbitrarily high values of $l$ (as needed for analytically implementing the mode-sum regularization procedure). Fortunately, for each pre-decided PN accuracy of the final result, we do not need to use the full power of the hypergeometric series expansions (33), (34). For instance, if we are interested in having $4 \mathrm{PN}$ accuracy for $h_{k k}^{R}$, the work of Ref. [23] has shown that, at the 4PN level, the subtle (formally infra-red divergent) mixing of near-zone and wave-zone effects only occurs through mass-type quadrupolar $(l=2)$ couplings. This indicates that, at the $4 \mathrm{PN}$ level, the full power of the hypergeometric series expansions is only needed to correctly get the $l=2$ contribution to $h_{k k}^{(l, m)}$, and that a usual PN expansion of the RWZ equations is accurate enough to evaluate the $l \geq 3$ contributions. On the other hand, if one aims at a higher PN accuracy, one needs to apply the MST technology to correspondingly higher values of $l$. Actually, the main role of the MST expansions is to correctly capture the transition between the near-zone and the wavezone, i.e., within our context, to correctly incorporate all tail effects in the near-zone metric perturbation $h_{\mu \nu}\left(x^{\lambda}\right)$ that we use to compute $h_{k k}(x)$. On the one hand, we know [23, 24] that these tail effects enter the near-zone metric as hereditary modifications of radiation reaction. On the other hand, we know that the PN order at which (leading-order) radiation effects enter the dynamics increases with the multipolarity $l$, and also depends on the even/odd character of the considered multipole. More precisely, from the study of radiation reaction linked to higher multipoles in Ref. [35], one knows that mass-type (i.e. even) multipoles of degree $l$ enter radiation reaction at the PN order $1 / c^{2 l+1}$, while spin-type (i.e. odd) multipoles of degree $l$ enter radiation reaction at the $\mathrm{PN}$ order $1 / c^{2 l+3}$. Tail effects linked to the total mass of the system then modify in an hereditary way these leading-order effects by adding corrections proportional to $G M / c^{3}$ and its higher powers. In other words, the IR-delicate hered- itary effects (for which we need MST technology) arise, from each multipole, at the following PN orders: (i) from $l=2$ even, at orders $1 / c^{5} \times\left(1 / c^{3}+1 / c^{6}+1 / c^{9}+\cdots\right)$ (i.e. $4 \mathrm{PN}, 5.5 \mathrm{PN}, 7 \mathrm{PN}$, etc); (ii) from $l=2$ odd, at orders $1 / c^{7} \times\left(1 / c^{3}+1 / c^{6}+1 / c^{9}+\cdots\right)$ (i.e. $5 \mathrm{PN}, 6.5 \mathrm{PN}$, $8 \mathrm{PN}$, etc); (ii) from $l=3$ even, at orders $1 / c^{7} \times\left(1 / c^{3}+\right.$ $\left.1 / c^{6}+1 / c^{9}+\cdots\right)$ (i.e. $5 \mathrm{PN}, 6.5 \mathrm{PN}, 8 \mathrm{PN}$, etc); (iii) from $l=3$ odd, at orders $1 / c^{9} \times\left(1 / c^{3}+1 / c^{6}+1 / c^{9}+\cdots\right)$ (i.e. $6 \mathrm{PN}, 7.5 \mathrm{PN}, 9 \mathrm{PN}$, etc); from $l=4$ even, at orders $1 / c^{9} \times\left(1 / c^{3}+1 / c^{6}+1 / c^{9}+\cdots\right)$ (i.e. $6 \mathrm{PN}, 7.5 \mathrm{PN}, 9 \mathrm{PN}$, etc); and so on. As our aim is to reach the $6 \mathrm{PN}$ accuracy, we see that it is sufficient to use hypergeometric series up to $l=4$ (even). For higher values of $l$ (and, actually, also for the odd, $l=4$ contribution) it must be sufficient to use PN-type solutions of the RW equations for $X$. In addition, the reasoning above shows that hypergeometric expansions should only be needed to get right the "up" part of the Green's function, which is the part which incorporates the transition between the near zone and the wave zone. [The "in" part of the Green's function, related to the matching between the near zone and the horizon, is not expected to influence the near zone metric until $4 \mathrm{PN}$ levels beyond radiation reaction [36], i.e. terms of order $1 / c^{5} \times 1 / c^{8}=1 / c^{13}$ (see Sec. 3.4 of Ref. [37]); moreover these terms are of radiative nature, and will start modifying the conservative effects we are considering only at a strictly higher order, i.e. strictly beyond the $6.5 \mathrm{PN}$ level.] We have explicitly checked the correctness of this expectation by studying in detail the difference between the exact expansions (33), (34) and their PN counterparts.

\section{E. Expanding the hypergeometric-series "in" and "up" homogeneous solutions in powers of $\eta:=1 / c$}

In this work we shall denote the basic PN expansion parameter as $\eta:=1 / c$. [We use the Greek letter $\eta$ because we have followed MST and other Japanese authors in using $\epsilon$ to denote $2 G M \omega / c^{3}$. Note that $\epsilon$ is of PN order $\eta^{3}$.] The PN expansion parameter $\eta:=1 / c$ has a double meaning: it keeps track both of the near-zone expansion (in powers of $\omega r / c$ ), and of the weak-field expansion (in powers of $\left.G M /\left(r c^{2}\right)\right)$. The expansions of $X_{l \omega}^{\text {in }}$ and $X_{l \omega}^{\text {up }}$ in powers of $\eta$ will have the general form

$$
\begin{aligned}
& X_{l \omega}^{\mathrm{in}}(r)=\tilde{c}_{\mathrm{in}} r^{l+1} \sum_{k=0}^{k_{\max }} A_{k}^{\mathrm{in}(l)} \eta^{k}, \\
& X_{l \omega}^{\mathrm{up}}(r)=\tilde{c}_{\mathrm{up}} r^{-l} \sum_{k=0}^{k_{\max }} A_{k}^{\mathrm{up}(l)} \eta^{k},
\end{aligned}
$$

where $\tilde{c}_{\text {in }}$ and $\tilde{c}_{\text {up }}$ are some normalization coefficients, where $A_{0}^{\text {in }(l)}=1=A_{0}^{\text {up }(l)}$, and where the logarithmic dependence in $\eta$ is absorbed in the expansion coefficients $A_{k}$. We shall consider the $A_{k}$ 's as functions of the two 
quantities $X_{1}=G M / r$ (linked to the weak-field expansion), and $\sqrt{X_{2}}:=\omega r$ (linked to the near-zone expansion). [The auxiliary arguments $X_{1}, X_{2}$ should not be confused with the dependent variables $X_{l \omega}^{\text {in }}, X_{l \omega}^{\text {up }}$.] Modulo some logarithmic dependence in $r$, the $A_{k}$ 's are polynomials in $X_{1}$ and $\sqrt{X_{2}}$.

Let us indicate the structure of the $\eta$ expansion of the crucial hypergeometric up solutions (34) for the particular case $l=2$. We derived similar explicit results for $l=3$ and 4 . In all cases we have extended the expansion up to $k_{\max }=12$, corresponding to the (fractional) $6 \mathrm{PN}$ level. When using $c_{\mathrm{up}}=c^{3}=\eta^{-3}$ in the definition of $C_{(\mathrm{up})}^{\nu}(z)$ in Eq. (34), the $\eta$ expansion of the hypergeometric "up" solution for $l=2, X_{(\text {up })}^{H G, l=2}$, reads

$$
X_{(\text {up })}^{H G, l=2}=-\frac{i}{16 \omega^{2} r^{2}} \sum_{k=0}^{12} A_{k}^{\mathrm{up}(H G, l=2)} \eta^{k}
$$

where HG stands for "hypergeometric" and the explicit values of the coefficients $A_{k}^{\text {up }}$ read

$$
\begin{aligned}
& A_{0}^{\text {up }(H G, l=2)}=1, \quad A_{1}^{\text {up }(H G, l=2)}=0, \\
& A_{2}^{\text {up }(H G, l=2)}=\frac{1}{6} X_{2}+\frac{5}{3} X_{1},
\end{aligned}
$$

$$
\begin{aligned}
& A_{3}^{\text {up }(H G, l=2)}=\left(6 i \gamma-2 \pi-\frac{43}{6} i\right) X_{1} \sqrt{X_{2}} \\
& A_{4}^{\text {up }(H G, l=2)}=\frac{7}{6} X_{1} X_{2}+\frac{1}{24} X_{2}^{2}+\frac{20}{7} X_{1}^{2} \\
& A_{5}^{\text {up }(H G, l=2)}=\left[\left(10 i \gamma-\frac{10}{3} \pi-\frac{215}{18} i\right) X_{1}^{2}+\left(-\frac{43}{36} i+i \gamma-\frac{1}{3} \pi\right) X_{1} X_{2}+\frac{1}{45} i X_{2}^{2}\right] \sqrt{X_{2}} \\
& A_{6}^{\text {up }(H G, l=2)}=5 X_{1}^{3}+\left(-18 \gamma^{2}+\frac{7}{3} \pi^{2}-12 i \pi \gamma-\frac{272551}{8820}+\frac{214}{105} \ln (2 \omega r \eta)+\frac{4729}{105} \gamma\right. \\
& \left.+\frac{43}{3} i \pi\right) X_{1}^{2} X_{2}+\frac{7}{24} X_{1} X_{2}^{2}-\frac{1}{144} X_{2}^{3} \\
& A_{7}^{\mathrm{up}(H G, l=2)}=\left[\left(-\frac{430}{21} i+\frac{120}{7} i \gamma-\frac{40}{7} \pi\right) X_{1}^{3}+\left(-\frac{301}{36} i-\frac{7}{3} \pi+7 i \gamma\right) X_{2} X_{1}^{2}\right. \\
& \left.+\left(-\frac{1}{12} \pi-\frac{43}{144} i+\frac{1}{4} i \gamma\right) X_{2}^{2} X_{1}-\frac{1}{630} i X_{2}^{3}\right] \sqrt{X_{2}} \\
& A_{8}^{\text {up }(H G, l=2)}=\frac{80}{9} X_{1}^{4}+\left(-20 i \pi \gamma+\frac{215}{9} i \pi+\frac{4729}{63} \gamma-30 \gamma^{2}+\frac{35}{9} \pi^{2}-\frac{1477681}{26460}\right. \\
& \left.+\frac{214}{63} \ln (2 \omega r \eta)\right) X_{2} X_{1}^{3}+\left(-\frac{76169}{17640}+\frac{7}{18} \pi^{2}+\frac{43}{18} i \pi-2 i \pi \gamma\right. \\
& \left.+\frac{4729}{630} \gamma-3 \gamma^{2}+\frac{107}{315} \ln (2 \omega r \eta)\right) X_{2}^{2} X_{1}^{2}+\left(\frac{3547}{10800}-\frac{4}{45} \ln (2 \omega r \eta)-\frac{2}{9} \gamma\right) X_{2}^{3} X_{1}+\frac{1}{3456} X_{2}^{4} \\
& A_{9}^{\text {up }(H G, l=2)}=\left[\left(-10 \pi-\frac{215}{6} i+30 i \gamma\right) X_{1}^{4}+\left(-2 \pi^{3}-36 i \gamma^{3}+\frac{4943}{35} i \gamma^{2}+\frac{428}{35} i \gamma \ln (2 \omega r \eta)\right.\right. \\
& +\frac{1691881}{17640} i-8 i \zeta(3)+14 i \pi^{2} \gamma+\frac{272551}{4410} \pi-\frac{4601}{315} i \ln (2 \omega r \eta)-\frac{428}{105} \pi \ln (2 \omega r \eta)+36 \gamma^{2} \pi \\
& \left.-\frac{9458}{105} \pi \gamma-\frac{882067}{4410} i \gamma-\frac{9251}{630} i \pi^{2}\right) X_{2} X_{1}^{3}+\left(\frac{7}{4} i \gamma-\frac{7}{12} \pi-\frac{301}{144} i\right) X_{2}^{2} X_{1}^{2} \\
& \left.+\left(\frac{121}{3360} i-\frac{1}{24} i \gamma+\frac{1}{72} \pi\right) X_{2}^{3} X_{1}+\frac{1}{22680} i X_{2}^{4}\right] \sqrt{X_{2}}
\end{aligned}
$$




$$
\begin{aligned}
& A_{10}^{\mathrm{up}(H G, l=2)}=16 X_{1}^{5}+\left(\frac{20}{3} \pi^{2}-\frac{240}{7} i \pi \gamma+\frac{860}{21} i \pi-\frac{220798}{2205}-\frac{360}{7} \gamma^{2}+\frac{856}{147} \ln (2 \omega r \eta)+\frac{18916}{147} \gamma\right) X_{2} X_{1}^{4} \\
& +\left(\frac{107}{45} \ln (2 \omega r \eta)-21 \gamma^{2}+\frac{301}{18} i \pi+\frac{4729}{90} \gamma-14 i \pi \gamma-\frac{33862}{945}+\frac{49}{18} \pi^{2}\right) X_{2}^{2} X_{1}^{3} \\
& +\left(-\frac{45901}{70560}+\frac{43}{72} i \pi+\frac{107}{1260} \ln (2 \omega r \eta)+\frac{4729}{2520} \gamma-\frac{1}{2} i \pi \gamma-\frac{3}{4} \gamma^{2}+\frac{7}{72} \pi^{2}\right) X_{2}^{3} X_{1}^{2} \\
& +\left(\frac{1}{63} \gamma-\frac{108721}{4233600}+\frac{2}{315} \ln (2 \omega r \eta)\right) X_{2}^{4} X_{1}-\frac{1}{172800} X_{2}^{5} \\
& A_{11}^{\mathrm{up}(H G, l=2)}=\left[\left(\frac{160}{3} i \gamma-\frac{1720}{27} i-\frac{160}{9} \pi\right) X_{1}^{5}+\left(\frac{428}{21} i \gamma \ln (2 \omega r \eta)-60 i \gamma^{3}+60 \gamma^{2} \pi+\frac{1477681}{13230} \pi+\frac{70}{3} i \pi^{2} \gamma-\frac{10}{3} \pi^{3}\right.\right. \\
& -\frac{428}{63} \pi \ln (2 \omega r \eta)-\frac{9458}{63} \pi \gamma+\frac{4943}{21} i \gamma^{2}-\frac{9251}{378} i \pi^{2}-\frac{40}{3} i \zeta(3)+\frac{30320033}{158760} i-\frac{4601}{189} i \ln (2 \omega r \eta) \\
& \left.-\frac{4755113}{13230} i \gamma\right) X_{2} X_{1}^{4}+\left(\frac{76169}{8820} \pi+\frac{3181751}{317520} i-\frac{4729}{315} \pi \gamma+6 \gamma^{2} \pi-\frac{1}{3} \pi^{3}-6 i \gamma^{3}-\frac{149987}{5292} i \gamma-\frac{4}{3} i \zeta(3)\right. \\
& \left.+\frac{214}{105} i \gamma \ln (2 \omega r \eta)-\frac{4601}{1890} i \ln (2 \omega r \eta)+\frac{7}{3} i \pi^{2} \gamma+\frac{4943}{210} i \gamma^{2}-\frac{214}{315} \pi \ln (2 \omega r \eta)-\frac{9251}{3780} i \pi^{2}\right) X_{2}^{2} X_{1}^{3} \\
& +\left(\frac{8}{45} \pi \gamma+\frac{932}{1575} i \ln (2 \omega r \eta)-\frac{8}{15} i \gamma \ln (2 \omega r \eta)-\frac{3532427}{2268000} i-\frac{14}{15} i \gamma^{2}-\frac{14501}{37800} \pi+\frac{6457}{2520} i \gamma\right. \\
& \left.+\frac{8}{45} \pi \ln (2 \omega r \eta)-\frac{1}{135} i \pi^{2}\right) X_{2}^{3} X_{1}^{2}+\left(-\frac{389}{311040} i-\frac{1}{1728} \pi+\frac{1}{576} i \gamma\right) X_{2}^{4} X_{1} \\
& \left.-\frac{1}{1496880} i X_{2}^{5}\right] \sqrt{X_{2}} \\
& A_{12}^{\mathrm{up}(H G, l=2)}=\frac{320}{11} X_{1}^{6}+\left(\frac{215}{3} i \pi-60 i \pi \gamma-\frac{4790743}{26460}+\frac{214}{21} \ln (2 \omega r \eta)+\frac{35}{3} \pi^{2}-90 \gamma^{2}+\frac{4729}{21} \gamma\right) X_{2} X_{1}^{5} \\
& +\left(-\frac{420397183}{661500} \gamma-\frac{5164}{105} \zeta(3)-\frac{7469477}{132300} \pi^{2}+\frac{121}{90} \pi^{4}+\frac{14243011}{22050} \gamma^{2}\right. \\
& +\frac{965664058429471}{4453225938000}-\frac{19861979}{330750} \ln (2 \omega r \eta)-\frac{10314}{35} \gamma^{3}+54 \gamma^{4}+\frac{22898}{11025} \ln ^{2}(2 \omega r \eta) \\
& +\frac{1012006}{11025} \gamma \ln (2 \omega r \eta)+\frac{29251}{315} \pi^{2} \gamma-\frac{1691881}{8820} i \pi+\frac{359}{35} i \pi^{3}-\frac{1284}{35} \gamma^{2} \ln (2 \omega r \eta) \\
& +\frac{214}{45} \ln (2 \omega r \eta) \pi^{2}+48 \zeta(3) \gamma-42 \pi^{2} \gamma^{2}-\frac{856}{35} i \pi \gamma \ln (2 \omega r \eta)+\frac{882067}{2205} i \pi \gamma \\
& \left.+72 i \pi \gamma^{3}-12 i \pi^{3} \gamma+16 i \pi \zeta(3)-\frac{9886}{35} i \gamma^{2} \pi+\frac{9202}{315} i \pi \ln (2 \omega r \eta)\right) X_{2}^{2} X_{1}^{4} \\
& +\left(\frac{4729}{360} \gamma+\frac{49}{72} \pi^{2}-\frac{100693}{12960}-\frac{7}{2} i \pi \gamma+\frac{301}{72} i \pi+\frac{107}{180} \ln (2 \omega r \eta)-\frac{21}{4} \gamma^{2}\right) X_{2}^{3} X_{1}^{3} \\
& +\left(\frac{103}{2520} \ln (2 \omega r \eta)-\frac{824923}{6350400}+\frac{1}{8} \gamma^{2}-\frac{7}{432} \pi^{2}-\frac{43}{432} i \pi-\frac{883}{5040} \gamma+\frac{1}{12} i \pi \gamma\right) X_{2}^{4} X_{1}^{2} \\
& +\left(-\frac{1}{5670} \ln (2 \omega r \eta)+\frac{170659}{228614400}-\frac{1}{2268} \gamma\right) X_{2}^{5} X_{1}+\frac{1}{14515200} X_{2}^{6} \text {. }
\end{aligned}
$$

Note that this "up" solution contains logarithmic terms, $\quad \ln (2 \omega r \eta)$, starting at the $3 \mathrm{PN}$ level (i.e. $\left.A_{6}^{\text {up }}\right)$, and even 
squared logarithms, $(\ln (2 \omega r \eta))^{2}$, at the $6 \mathrm{PN}$ level $A_{12}^{\text {up }}$. These logarithms have various physical meanings: some are gauge effects, some are related to far-zone effects and enter the relation between algorithmic multipole moments and source variables 1, some (with imaginary coefficients) are linked to tail modifications of radiationreaction effects, and some are linked to tail modifications of conservative effects. For instance, the term $-\frac{4}{45} \ln (2 \omega r \eta) X_{2}^{3} X_{1}$, which contains a factor $M \omega^{6}$, is encoding the conservative part of the $4 \mathrm{PN}$ near-zone hereditary term discussed in [19, 22, 23]. Our computation of the quantity $h_{k k}$ will automatically select, among all these logarithmic contributions, the gauge-invariant conservative ones.

As for the ingoing hypergeometric solution $X_{l \omega}^{(\mathrm{in})}(r)$ we found that, modulo an inessential constant prefactor, it is correctly evaluated by solving the corresponding homogeneous RW equation by a formal PN scheme, of the type we explain next.

F. Deriving sufficiently accurate homogeneous PN-expanded solutions for general multipole degree $l$

The hypergeometric solutions (33), (34) automatically incorporate all the physically correct boundary conditions, but are difficult to evaluate for a general multipole degree $l$. By contrast, for generic $l$, one can look for solutions of the homogeneous RW equations $\mathcal{L}_{(\mathrm{RW})}^{(r)} X_{(\mathrm{in})}(r)=0=\mathcal{L}_{(\mathrm{RW})}^{(r)} X_{(\mathrm{up})}(r)$ satisfied by both $X_{l \omega}^{(\mathrm{in})}$ and $X_{l \omega}^{(\mathrm{up})}$ in the form of a usual PN expansion, i.e.

$$
\begin{aligned}
X_{l \omega}^{\mathrm{in}(\mathrm{PN})}(r) & =r^{l+1}\left[1+\eta^{2} A_{2}^{(P N, l)}+\eta^{4} A_{4}^{(P N, l)}\right. \\
& \left.+\eta^{6} A_{6}^{(P N, l)}+\ldots+\eta^{12} A_{12}^{(P N, l)}+\ldots\right]
\end{aligned}
$$

$$
\begin{aligned}
X_{l \omega}^{\mathrm{up}(\mathrm{PN})}(r) & =r^{-l}\left[1+\eta^{2} A_{2}^{(P N,-l-1)}+\eta^{4} A_{4}^{(P N,-l-1)}\right. \\
& \left.+\eta^{6} A_{6}^{(P N,-l-1)}+\ldots+\eta^{12} A_{12}^{(P N,-l-1)}+\ldots\right]
\end{aligned}
$$

As the coefficients entering the RW operator $\mathcal{L}_{(\mathrm{RW})}^{(r)}$ (16) are polynomial in $G M / c^{2}=M \eta^{2}$ and $\omega^{2} / c^{2}=\omega^{2} \eta^{2}$, one can look for PN expansion coefficients $A_{2}^{(P N, l)}, A_{4}^{(P N, l)}, \ldots$ (restricted, as indicated in Eqs. (42), (43), to even powers of $\eta=1 / c$, i.e. to even values of the index $k$ ) in the form of polynomials in $X_{1}=G M / r, X_{2}=(\omega r)^{2}$ with $l$-dependent coefficients. One finds that it is indeed possible to find such solutions. The first three coefficients of such PN-expanded solutions

1 This is the case of the $\ln \eta$ appearing at $3 \mathrm{PN}$; see [23, 38]. read

$$
\begin{aligned}
A_{0}^{\text {in }(P N, l)}= & 1 \\
A_{2}^{\text {in }(P N, l)}= & -\frac{(l-2)(l+2)}{l} X_{1}-\frac{1}{2(2 l+3)} X_{2} \\
A_{4}^{\text {in }(P N, l)}= & \frac{(l-2)(l-3)(l+2)(l+1)}{(-1+2 l) l} X_{1}^{2} \\
& +\frac{\left(l^{3}-5 l^{2}-14 l-12\right)}{2(l+1)(2 l+3) l} X_{2} X_{1} \\
& +\frac{1}{8(2 l+5)(2 l+3)} X_{2}^{2} \\
A_{6}^{\text {in }(P N, l)}= & -\frac{(l-2)(l-3)(l-4)(l+2)(l+1)}{3(-1+2 l)(l-1)} X_{1}^{3} \\
& -\frac{2\left(15 l^{4}+30 l^{3}+28 l^{2}+13 l+24\right)}{(-1+2 l)(2 l+1)(l+1) l(2 l+3)} \times \\
& \times X_{1}^{2} X_{2} \ln (r / R) \\
& -\frac{\left(3 l^{4}-27 l^{3}-154 l^{2}-220 l-120\right)}{24(l+1) l(2 l+5)(l+2)(2 l+3)} X_{1} X_{2}^{2} \\
& -\frac{1}{48(2 l+5)(2 l+7)(2 l+3)} X_{2}^{3}
\end{aligned}
$$

As illustrated here for the coefficient $A_{6}^{(P N, l)}$, it is necessary to go beyond a purely polynomial structure in $X_{1}=$ $G M / r$ and $X_{2}=(\omega r)^{2}$, and to introduce, in a few terms, a logarithmic dependence $A_{2 k, p, q}^{(P N, l) \log } \ln (r / R) X_{1}^{p} X_{2}^{q}$ in the coefficients $A_{2 k}^{(P N, l)}$ entering the possible form of the PN expansion of $X_{l \omega}^{\text {in }}$. Such logarithmic terms must be included in $A_{6}^{(P N, l)}, A_{8}^{(P N, l)}, A_{10}^{(P N, l)}$, and $A_{12}^{(P N, l)}$. In addition, as indicated in Eq. (43) above, the coefficients entering the up PN solution $X_{l \omega}^{\mathrm{up}(\mathrm{PN})}$, Eq. (43)), are formally obtained from the $A_{2 k}^{(l)}$ coefficients entering the in PN solution Eq. (42) simply by replacing $l$ by $-l-1$. However, this formal replacement rule sometimes generates poles $\propto(l-n)^{-1}$ where $n$ is a positive integer. Such poles correspond to a denominator of the form $(l+n+1)^{-1}$ in some coefficient $A_{2 k}^{(P N, l)}$ in $X_{l \omega}^{\text {in(PN) }}$. For instance, the $4 \mathrm{PN}$ "in" coefficient $A_{8}^{(P N, l)}$ contains the following term

$$
+\frac{\left(5 l^{5}-60 l^{4}-645 l^{3}-1788 l^{2}-1928 l-840\right)}{240 l(l+3)(2 l+3)(l+1)(2 l+5)(2 l+7)(l+2)} X_{1} X_{2}^{3}
$$

This term (which is $\propto X_{1} X_{2}^{3}$ ) contains a factor $l+3$ in the denominator that will generate a denominator $l-2$ in the corresponding $4 \mathrm{PN}$-level term $A_{8}^{(P N,-l-1)}$ of $X_{l \omega}^{\mathrm{up}(\mathrm{PN})}$. When considering the integer value $l=2$, one must replace the ill-defined term $\propto X_{1} X_{2}^{3} /(l-2)$ in $X_{l \omega}^{\mathrm{up}(\mathrm{PN})}$ by a new logarithmic term $\propto X_{1} X_{2}^{3} \ln (r / R)$. Similar additional logarithms in the "up" solution also arise (for the multipole $l=2$ ) from the "in" coefficients $A_{10}^{(P N, l)}$ and $A_{12}^{(P N, l)}$. Analogous additional logarithms also arise for $l=3$ from $A_{10}^{(P N, l)}$ and $A_{12}^{(P N, l)}$, and for $l=4$ from $A_{12}^{(P N, l)}$. 
The so generated PN solutions depend on the arbitrary scale $R$ (entering the two sorts of logarithms discussed above). Moreover, these formal solutions do not correctly incorporate the retarded-type boundary condition included in the hypergeometric solutions (33), (34). [For instance, the contributions proportional to odd powers of $\eta$ in (33) come from the retarded nature of the "up" solution.] However, we have checked (by comparing the structure of the hypergeometric expansions to that of the simpler PN expansions) that, for $l \geq 5$, they give the same value for $h_{k k}$ (up to $6 \mathrm{PN}$ ). [In particular, the arbitrary logarithmic scale $R$ drops out in the $6 \mathrm{PN}$ calculation of $h_{k k}$.] Moreover, for $l=2, l=3$ and $l=4$, where one must use the hypergeometric-type solution for $X_{l \omega}^{(\text {up) }}(r)$, it would be enough to use the PN-one for $X_{l \omega}^{(\text {in) }}(r)$. Indeed, an explicit calculation shows that they are proportional to each other (up to $\eta^{12}$ included). For instance, we found

$$
X_{(\mathrm{in})}^{H G, l=2}=\frac{i e^{\psi_{2}}}{384 M^{4} \omega} X_{(\mathrm{in})}^{P N, l=2}
$$

where

$$
\begin{aligned}
\psi_{2}= & -\frac{94}{35} i M \omega \eta^{3}-\left[\frac{24197}{4900}+\frac{214}{105} \ln \left(\frac{R}{2 M \eta^{2}}\right)\right] \omega^{2} M^{2} \eta^{6} \\
& -\left(\frac{1099309}{385875}+\frac{428}{315} \pi^{2}\right) i M^{3} \omega^{3} \eta^{9} \\
& +\left[-\frac{832550660525191}{44532259380000}+\frac{1712}{105} \zeta(3)\right. \\
& \left.+\frac{45796}{33075} \pi^{2}-\frac{3390466}{1157625} \ln \left(\frac{R}{2 M \eta^{2}}\right)\right] M^{4} \omega^{4} \eta^{12}
\end{aligned}
$$

and $R$ is the arbitrary (constant) scale entering the logarithmic terms in the $\mathrm{PN}$ solution. The $6 \mathrm{PN}$-accurate identity (modulo an overall factor) between $X_{(\text {in) }}^{H G, l}$ and $X_{(\mathrm{in})}^{P N, l}$ follows from the result of [36, 37] that the horizon boundary condition does not influence the PN-expanded, near-zone metric until terms of order $O\left(\eta^{13}\right)$.

\section{G. Summing over $m$}

When inserting the results (38), (39), (42), (43) in the expressions (of the type (29), (30)) giving $h_{k k, l m}$ we get explicit results which depend both on $l$ and on $m$ via the $m$-dependent value of $\omega=m \Omega$. Indeed, $\omega$ appeared from the start in the RW equations, and thereby in their solutions; e.g., note that the parameter $\epsilon=2 G M \omega / c^{3}$ pervasively entering the hypergeometric expansions (33), (34) (and the $l$-deformed parameter $\nu$, Eq. (35) is proportional to $m$. The summation over $m$ in Eq. (12) then generates finite sums most of which are of the form

$$
S_{N, l}=\sum_{m=-l}^{+l} m^{N}\left|Y_{l m}(\pi / 2,0)\right|^{2},
$$

or

$$
S_{N, l}^{\prime}=\sum_{m=-l}^{+l} m^{N}\left|\partial_{\theta} Y_{l m}(\pi / 2,0)\right|^{2} .
$$

These sums vanish when the (non negative) integer $N$ is odd, and can be expressed as polynomials in $l$ when $N$ is even, thanks to the results of the Japanese relativity school; see Appendix F in [16]. Actually, we needed to go beyond the explicit results given in the latter reference: indeed, we needed $S_{N, l}$ up to $N=12$, and $S_{N, l}^{\prime}$ up to $N=10$. The explicit values of the above sums for $l=2$ and 3 are:

$$
\begin{aligned}
S_{N, 2} & =\sum_{m=-2}^{2} m^{N}\left|Y_{2 m}(\pi / 2,0)\right|^{2} \\
& =\frac{15}{32 \pi}\left[(-2)^{N}+2^{N}\right] \\
S_{N, 3} & =\sum_{m=-3}^{3} m^{N}\left|Y_{3 m}(\pi / 2,0)\right|^{2} \\
& =\frac{35}{64 \pi}\left[(-3)^{N}+3^{N}\right]+\frac{21}{64 \pi}\left[(-1)^{N}+1\right],
\end{aligned}
$$

and

$$
\begin{aligned}
S_{N, 2}^{\prime} & =\sum_{m=-2}^{+2} m^{N}\left|\partial_{\theta} Y_{2 m}(\pi / 2,0)\right|^{2} \\
& =\frac{15}{8 \pi}\left[(-1)^{N}+1\right] \\
S_{N, 3}^{\prime} & =\sum_{m=-3}^{+3} m^{N}\left|\partial_{\theta} Y_{3 m}(\pi / 2,0)\right|^{2} \\
& =\frac{105}{32 \pi}\left[(-2)^{N}+2^{N}\right] .
\end{aligned}
$$

In addition to the sums (48), (49), our 6PN-accurate calculation of $h_{k k}^{R}$ involved new, and more delicate, sums of the type

$$
S_{N, l}^{\mathrm{log}}=\sum_{m=-l}^{+l} m^{N} \ln (-i m)\left|Y_{l m}(\pi / 2,0)\right|^{2} .
$$

Our 6PN calculation involve such logarithmic sums for the $l$ values $l=2, l=3$ and $l=4$. For $l=2$ and $l=3$, we have for example

$$
\begin{aligned}
S_{N, 2}^{\log =} & \sum_{m=-2}^{+2} m^{N} \ln (-i m)\left|Y_{2 m}(\pi / 2,0)\right|^{2} \\
= & \frac{15}{32 \pi}\left(2^{N}+(-2)^{N}\right) \ln 2-\frac{15}{64} i\left(2^{N}-(-2)^{N}\right) \\
S_{N, 3}^{\log =} & \sum_{m=-3}^{+3} m^{N} \ln (-i m)\left|Y_{3 m}(\pi / 2,0)\right|^{2} \\
= & \frac{35}{64 \pi}\left(3^{N}+(-3)^{N}\right) \ln 3 \\
& -\frac{35}{128} i\left(3^{N}-(-3)^{N}\right)-\frac{21}{128} i\left(1-(-1)^{N}\right) .(53)
\end{aligned}
$$


Contrary to the non-logarithmic sums above, these logarithmic sums do not vanish for odd values of $N$. Separating the cases where $N$ is even or odd we have

$$
S_{2 N, 2}^{\log }=\frac{15}{16 \pi} 2^{2 N} \ln 2, \quad S_{2 N+1,2}^{\log }=-\frac{15}{32} i 2^{2 N+1}
$$

and

$$
\begin{aligned}
S_{2 N, 3}^{\log } & =\frac{35}{32 \pi} 3^{2 N} \ln 3 \\
S_{2 N+1,3}^{\log } & =-\frac{7 i}{64}\left(5 \cdot 3^{2 N+1}+3\right) .
\end{aligned}
$$

These sums explain the appearance of $\ln 2$ and $\ln 3$ in our final results. Note also that the squared logarithms that entered the $6 \mathrm{PN}$ "up" coefficients $A_{12}^{\text {up }(H G, l)}$ do not lead (at the $6 \mathrm{PN}$ order) to a conservative contribution involving a sum of the type (52) with $\ln (-i m)$ replaced by its square.

H. Subtracting $\tilde{D}_{0}$, and summing the series over the multipolar degree $l$

After having explicitly performed the summation over $m$, we need to subtract from each $l$-contribution the $u$ expansion of $\tilde{D}_{0}=(1-3 u) D_{0}$ (with Eq. (11)), namely

$$
\begin{aligned}
\tilde{D}_{0} & =2 u-\frac{13}{2} u^{2}+\frac{9}{32} u^{3}+\frac{83}{128} u^{4}+\frac{12361}{8192} u^{5} \\
& +\frac{116163}{32768} u^{6}+\frac{1867635}{32768} u^{7}+O\left(u^{8}\right) .
\end{aligned}
$$

We then obtain, according to Eq. (12), an explicit expression for $h_{k k}^{R}$ given by a sum of the following type: (i) a few exact contributions for $l=0$ and $l=1$; (ii) the explicit contributions from $l=2, l=3$ and $l=4$ (expanded up to $\eta^{12}$ ); and, finally, (iii) an infinite series over $l \geq 4$. The latter series converges, and can be explicitly evaluated thanks to the fact that the PN-expanded RW solutions for generic values of $l$ can be written in terms of explicit rational functions of $l$. In other words, our calculation involves series of the type $\sum_{l>3} P_{n-2}(l) / Q_{n}(l)$ with (complicated) polynomials of degree $n-2$ and $n$ respectively. For instance, at the $4 \mathrm{PN}$ level we have a polynomial $Q_{14}(l)$ of degree 14 in the denominator. [However, $Q_{14}(l)$ is factorizable in ten simpler factors $\sim(a l+b)^{k}$ with $k=1$ or 2 and $a, b \in \mathbb{Z}$.] In the course of this calculation, one checks that $\tilde{D}_{0}$ does precisely subtract the $l \rightarrow \infty$ piece in the corresponding unsubtracted form of $h_{k k}^{(l)}$, which, e.g., involves at the $4 \mathrm{PN}$ level a rational fraction of the type $P_{14}(l) / Q_{14}(l)$.

The convergent series entering our calculation can be evaluated (after decomposing it in partial fractions in $l$ ) in terms of the well-known Euler series $\zeta(2)=\sum_{l} 1 /(l+$ $1)^{2}=\pi^{2} / 6$, and $\zeta(4)=\sum_{l} 1 /(l+1)^{4}=\pi^{4} / 90$.
This finally leads to our $6 \mathrm{PN}$-accurate result

$$
\begin{aligned}
h_{k k}^{R}= & -2 u+5 u^{2}+\frac{5}{4} u^{3}+\left(-\frac{1261}{24}+\frac{41}{16} \pi^{2}\right) u^{4} \\
+ & \left(\frac{157859}{960}-\frac{256}{5} \gamma-\frac{128}{5} \ln (u)-\frac{512}{5} \ln (2)\right. \\
& \left.-\frac{2275}{256} \pi^{2}\right) u^{5} \\
+ & \left(\frac{284664301}{201600}+\frac{28016}{105} \gamma+\frac{14008}{105} \ln (u)\right. \\
& \left.+\frac{63472}{105} \ln (2)-\frac{246367}{1536} \pi^{2}-\frac{486}{7} \ln (3)\right) u^{6} \\
& -\frac{27392}{525} \pi u^{13 / 2} \\
+ & \left(-\frac{413480}{567} \ln (2)+\frac{5044}{405} \ln (u)+\frac{10088}{405} \gamma\right. \\
& +\frac{22848244687}{7257600}+\frac{4617}{7} \ln (3) \\
& \left.+\frac{2800873}{131072} \pi^{4}-\frac{608698367}{884736} \pi^{2}\right) u^{7}+o\left(u^{7}\right) .(57
\end{aligned}
$$

\section{6PN-ACCURATE COMPUTATION OF THE EOB RADIAL POTENTIAL $A(u ; \nu)$ AT LINEAR ORDER IN $\nu$}

Let us now translate our results on Deweiler's gaugeinvariant function $h_{k k}^{R}(u)$ into the crucial radial interaction potential $A\left(r ; m_{1}, m_{2}\right)$ entering the EOB framework. The potential $A\left(r ; m_{1}, m_{2}\right)$ is a gauge-invariant function which enters the EOB formalism [2 5]. It is a useful generalization of the well-known Schwarzschild potential $A^{S}(r)=1-2 G M / c^{2} r$. The EOB formalism maps the conservative dynamics of a (non spinning) two-body system $\left(m_{1}, m_{2}\right)$ onto the geodesic dynamics of one body of mass $\mu=m_{1} m_{2} /\left(m_{1}+m_{2}\right)$ in a stationary and spherically symmetric "effective" metric,

$$
\begin{aligned}
d s_{\mathrm{eff}}^{2} & =-A\left(r ; m_{1}, m_{2}\right) c^{2} d t^{2} \\
& +B\left(r ; m_{1}, m_{2}\right) d r^{2}+r^{2}\left(d \theta^{2}+\sin ^{2} \theta d \varphi^{2}\right),
\end{aligned}
$$

together with post-geodesic corrections described by a function $Q\left(r, p_{r}, p_{\varphi} ; m_{1}, m_{2}\right)$ which is, at least, quartic in the (EOB) radial momentum $p_{r}$ [4]. The latter (gaugefixing) restriction ensures that the gauge-invariant dynamics of the sequence of circular orbits is fully encoded in the sole radial potential $A\left(r ; m_{1}, m_{2}\right)$ [7, 22, 28]. One can write the radial potential $A\left(r ; m_{1}, m_{2}\right)$ as a function of the two variables $u:=G M / c^{2} r$ and $\nu=\mu / M$ : $A\left(r ; m_{1}, m_{2}\right) \equiv A(u ; \nu)$.

From the analytical point of view, one can approach this function of two variables in two different, and complementary, ways: either by expanding it in powers of $u$, 
or in powers of $\nu$. The expansion in $u$ corresponds to the usual PN expansion, while the expansion in $\nu$ is a GSF expansion. In addition, one can also approach the radial potential $A(u ; \nu)$ by several types of numerical simulations: either full, three-dimensional numerical relativity simulations, or GSF numerical simulations. In this paper, we shall focus on analytical knowledge, without dealing with its comparison with numerical results.

Before discussing the new, higher PN contributions to $A(u ; \nu)$ that we shall derive below, let us recall the present $\mathrm{PN}$ knowledge of $A(u ; \nu)$. Its $\mathrm{PN}$ expansion is completely known only up to the $4 \mathrm{PN}$ level (included), i.e. up to terms of order $u^{5}$. At the $5 \mathrm{PN}$ level (i.e. $O\left(u^{6}\right)$ ), only the logarithmic contributions are so far known. At this order, the radial potential has the form

$$
\begin{aligned}
A(u ; \nu) & =1-2 u+\nu a_{3}(\nu) u^{3}+\nu a_{4}(\nu) u^{4} \\
& +\nu\left(a_{5}^{c}(\nu)+a_{5}^{\ln }(\nu) \ln u\right) u^{5} \\
& +\nu\left(a_{6}^{c}(\nu)+a_{6}^{\ln }(\nu) \ln u\right) u^{6}+o\left(u^{6}\right) .
\end{aligned}
$$

There is no $1 \mathrm{PN}-$ level contribution (i.e. $a_{2}(\nu)=0$ ). The value of the $2 \mathrm{PN}$-level coefficient, namely

$$
a_{3}(\nu)=2,
$$

was derived in [2] from the 2PN Delaunay Hamiltonian of [39]. The value of the $3 \mathrm{PN}-$ level coefficient, namely

$$
a_{4}(\nu)=\frac{94}{3}-\frac{41}{32} \pi^{2},
$$

was derived in [4] from the 3PN Hamiltonian of [40, 41]. The value of the $4 \mathrm{PN}$-level logarithmic coefficient, namely

$$
a_{5}^{\ln }(\nu)=\frac{64}{5}
$$

was derived in [7, 19, 22] from the results of Ref. 23]. The value of the non-logarithmic $4 \mathrm{PN}$-level coefficient in Eq. (59) was derived in [1], and found to be equal to (with $\gamma$ denoting Euler's constant)

$$
\begin{aligned}
a_{5}^{c}(\nu) & =a_{5}^{c 0}+\nu a_{5}^{c 1} \\
a_{5}^{c 0} & =-\frac{4237}{60}+\frac{2275}{512} \pi^{2}+\frac{256}{5} \ln 2+\frac{128}{5} \gamma \\
a_{5}^{c 1} & =-\frac{221}{6}+\frac{41}{32} \pi^{2} .
\end{aligned}
$$

Finally, at the 5PN level, only the logarithmic contribution $\nu a_{6}^{\ln }(\nu) \ln u u^{6}$ has been obtained [7, 19, 21], and found to be given by

$$
a_{6}^{\ln }(\nu)=-\frac{7004}{105}-\frac{144}{5} \nu
$$

In the present paper, we shall extend our analytical knowledge of $A(u ; \nu)$ to the $6 \mathrm{PN}$ level, but only for the terms linear in $\nu$. Our results are then most simply expressed in terms of the first coefficient function $a(u)$ of the GSF expansion of the radial potential $A(u ; \nu)$ :

$$
A(u ; \nu)=1-2 u+\nu a(u)+\nu^{2} a_{2}(u)+\nu^{3} a_{3}(u)+O\left(\nu^{4}\right) .
$$

The terms $\nu a(u)+\nu^{2} a_{2}(u)+\cdots$ represent (from the point of view of GSF theory) corrections to the test-mass limit (i.e. $A^{S}(u)=1-2 G M / c^{2} r=1-2 u$ ) coming from GSF effects. Note that, using the link (44), Refs. 7] and 28] gave numerical estimates of the EOB function $a(u)$ beyond the weak-field (PN) regime $u \ll 1$. In particular, Akcay et al. 28] gave accurate numerical representations of the function $a(u)$ over the interval $0<u<1 / 3$, and discovered the presence of a singularity near the "lightring" $u \rightarrow 1 / 3$.

Previous work has shown that remarkable cancellations occur in the EOB potential $A(u ; \nu)$ : while related functions (such as the energy-versus-frequency function $E(x ; \nu))$ generally contain, at each $\mathrm{PN}$ order, all the a priori possible powers of $\nu$, the $\mathrm{PN}$ expansion of $A(u ; \nu)$ is linear in $\nu$ up to the 3PN order (included) [2, 4]; in other words, the $O\left(\nu^{2}\right)$ term $a_{2}(u)$ in Eq. (65) starts only at the $4 \mathrm{PN}$ order $\sim u^{5}[1]$. The latter work also showed the presence of cancellations at the $4 \mathrm{PN}$ order; namely, the $u^{5}$ contribution to $A(u ; \nu)$ is no more than quadratic in $\nu$ (by contrast with, e.g., the energy-frequency function $E\left(G M \Omega / c^{3} ; \nu\right)$ which has $O\left(\nu^{3}\right)$ and $O\left(\nu^{4}\right)$ contributions [42]). In other words, the $O\left(\nu^{3}\right)$ term $a_{3}(u)$ in Eq. (65) does not start before the 5PN order $u^{6}$.] We shall further discuss these cancellations below.

Inserting our result Eq. (57) for $h_{k k}^{R}$ in the link (44), we determined the PN expansion of the $1 \mathrm{GSF}$ coefficient $a(u)$ up to the $6 \mathrm{PN}$ level, i.e.

$$
\begin{aligned}
a(u)= & a_{3} u^{3}+a_{4} u^{4}+a_{5}(\ln u) u^{5}+a_{6}(\ln u) u^{6} \\
& +a_{6.5} u^{13 / 2}+a_{7}(\ln u) u^{7}+o\left(u^{7}\right)
\end{aligned}
$$

The coefficients $a_{3}, \cdots, a_{6}(\ln u)$ here correspond to the $\nu \rightarrow 0$ limits of the PN-expansion coefficients entering Eq. (59) above; i.e. $a_{3}=a_{3}(0), a_{4}=a_{4}(0), a_{5}(\ln u)=$ $a_{5}^{c}(0)+a_{5}^{\ln }(0) \ln u, a_{6}(\ln u)=a_{6}^{c}(0)+a_{6}^{\ln }(0) \ln u$. For these coefficients, our results confirm the previous determinations of $a_{3}(0), a_{4}(0), a_{5}^{c}(0), a_{5}^{\ln }(0)$ and $a_{6}^{\ln }(0)$ recalled above, and extend them by providing the analytical values of $a_{6}^{c}(0), a_{6.5}$ and $a_{7}(\ln u)=a_{7}^{c}(0)+a_{7}^{\ln (0)} \ln u$. Namely, we found

$$
\begin{aligned}
a_{6}(\ln u)= & -\frac{1066621}{1575}-\frac{14008}{105} \gamma-\frac{7004}{105} \ln (u) \\
& -\frac{31736}{105} \ln (2)+\frac{246367}{3072} \pi^{2}+\frac{243}{7} \ln (3)(67 \mathrm{a}) \\
a_{6.5}= & +\frac{13696}{525} \pi, \quad(67 \mathrm{~b}) \\
a_{7}(\ln u)= & \frac{206740}{567} \ln (2)-\frac{2522}{405} \ln (u)-\frac{5044}{405} \gamma \\
& -\frac{1360201207}{907200}-\frac{4617}{14} \ln (3)-\frac{2800873}{262144} \pi^{4} \\
& +\frac{608698367}{1769472} \pi^{2} .
\end{aligned}
$$

Note that the transcendentality of the coefficients $a_{n}$ increases with $n$ : $a_{3}$ is rational; $a_{4}$ involves $\zeta(2)=\pi^{2} / 6 ; a_{5}$ involves $\zeta(2), \ln 2$ and $\gamma ; a_{6}$ involves $\zeta(2), \ln 2, \gamma$ and $\ln 3$; while $a_{7}$ involves $\zeta(2), \ln 2, \ln 3, \gamma$, and $\zeta(4)$. Formally, 
writing the Fokker action of the binary system in terms of classical Feynman-like diagrams [43] leads to integral expressions for the expansion coefficients of the radial potential $A(u, \nu)$ which are of the type called "periods" in mathematics. However, for circular orbits, these periods involve (non algebraic) helical world lines (which explains the appearance of Euler's constant $\gamma$ ).

\section{A. Binding energy of binary systems in terms of the orbital frequency}

The (main) EOB radial potential $A(u ; \nu)$ (which defines the $g_{00}(r)$ component of the effective metric entering the EOB formalism) completely describes the gaugeinvariant dynamics of circular orbits of binary systems. In particular, the potential $A(u ; \nu)$ allows one to give a simple parametric representation of the functional link between the total energy $H^{\text {tot }}$ of a binary system and the frequency of a circular orbit [3, 7, 22, 28]. Let us briefly recall this parametric representation, and use it to determine the functional relation between the energy and the orbital frequency (or, more conveniently, the dimensionless frequency parameter $\left.x=(M \Omega)^{2 / 3}\right)$.

Given the EOB potential $A(u ; \nu)$, the total energy $H^{\text {tot }}$ can be computed as an explicit function of $u$. Let us first define the functions

$$
\begin{gathered}
\tilde{A}(u ; \nu):=A(u ; \nu)+\frac{1}{2} u \partial_{u} A(u ; \nu), \\
\hat{H}_{\mathrm{eff}}(u ; \nu):=\frac{A(u ; \nu)}{\sqrt{\tilde{A}(u ; \nu)}}, \\
h(u ; \nu):=\sqrt{1+2 \nu\left(\hat{H}_{\mathrm{eff}}(u ; \nu)-1\right)} .
\end{gathered}
$$

In terms of this notation, the total energy of the circular binary (with $c=1)$ is simply $H^{\text {tot }}(u ; \nu)=M h(u ; \nu)$ so that the binding energy $E_{B}=H^{\text {tot }}-M$ reads

$$
\begin{aligned}
E_{B}(u ; \nu) & =M(h(u ; \nu)-1) \\
& =M\left(\sqrt{1+2 \nu\left(\hat{H}_{\mathrm{eff}}(u ; \nu)-1\right)}-1\right) .
\end{aligned}
$$

On the other hand, the dimensionless frequency parameter $x=(M \Omega)^{2 / 3}$ is given by the following function of

$$
x(u ; \nu)=u\left(\frac{-\frac{1}{2} \partial_{u} A(u ; \nu)}{h^{2}(u ; \nu)}\right)^{1 / 3} .
$$

Up to now we have made no approximation, so that Eqs. (71) and (72) yield an exact parametric representation of the functional link between $E_{B}$ and $x$. If we now consider the PN expansion of $A(u ; \nu)$ up to some $\mathrm{PN}$ level, we get corresponding $\mathrm{PN}$ expansions of $E_{B}(u)$ and $x(u)$. Inverting the latter expansion (which starts as $\left.x(u)=u+\frac{1}{3} \nu u^{2}+O\left(u^{3}\right)\right)$ to get $u$ in terms of $x$, we can straightforwardly obtain the $\mathrm{PN}$ expansion of the function relating $E_{B}$ to the frequency parameter $x$.

Let us start from the PN expansion of $A(u ; \nu)$ written up to the $6 \mathrm{PN}$ level, i.e.

$$
\begin{gathered}
A(u ; \nu)=1-2 u+\nu a_{3}(\nu) u^{3}+\nu a_{4}(\nu) u^{4}+\nu\left(a_{5}^{c}(\nu)\right. \\
\left.\quad+a_{5}^{\ln }(\nu) \ln (u)\right) u^{5}+\nu\left(a_{6}^{c}(\nu)+a_{6}^{\ln }(\nu) \ln (u)\right) u^{6} \\
+\nu a_{6.5}(\nu) u^{13 / 2}+\nu\left(a_{7}^{c}(\nu)+a_{7}^{\ln }(\nu) \ln (u)\right) u^{7}
\end{gathered}
$$

Here, we assumed that there are no extra terms that have not been detected by our $\nu$-linear computation at 5, 5.5 and $6 \mathrm{PN}$; for instance, we assume that there are no terms of the type $\nu^{2}(\ln u)^{2}$ in the higher PN coefficients $a_{n}(\nu, \ln u)$. Let us use in this expansion all the information we currently have; i.e., let us replace the known coefficients $a_{n}(\nu, \ln u)$ (up to $n=5$ ), as well as $a_{6}^{\ln }(\nu)$, by their known values (recalled at the beginning of this Section), and let us replace the coefficients which are only known to linear order in $\nu$ by general expressions of the type: $a_{6}^{c}(\nu)=a_{6}^{c}(0)+\nu a_{6}^{c 1}(\nu)$, $a_{6.5}(\nu)=a_{6.5}^{c}(0)+\nu a_{6.5}^{c 1}(\nu), a_{7}^{c}(\nu)=a_{7}^{c}(0)+\nu a_{7}^{c 1}(\nu)$, $a_{7}^{\ln }(\nu)=a_{7}^{\ln }(0)+\nu a_{7}^{\ln 1}(\nu)$,

where all the coefficients $a_{6}^{c}(0), a_{6.5}^{c}(0), a_{7}^{c}(0), a_{7}^{\ln }(0)$ have been determined above, and will be henceforth replaced by their explicit values. Note that we have allowed here for an a priori arbitrary $\nu$ dependence of the terms of order $O\left(\nu^{2}\right)$ that are not controlled by our present results. Actually, we expect that the $\nu$ dependence of these coefficients will be rather simple. See below.

With such a parametrization of our current knowledge (and of our remaining ignorance), we find that the coefficients in the $6 \mathrm{PN}$ expansion Eq. (6) of the function $E_{B}(x ; \nu)$ are given by the following expressions (in which we suppress, for notational clarity, the arguments $\nu$ in $a_{6}^{c 1}(\nu), a_{6.5}^{c 1}(\nu), a_{7}^{c 1}(\nu)$, and $a_{7}^{\ln 1}(\nu)$, and separate the logarithmic contributions as $e_{\mathrm{nPN}}(\nu, \ln x) \equiv$ $\left.e_{\mathrm{nPN}}^{c}(\nu)+e_{\mathrm{nPN}}^{\ln }(\nu) \ln x\right)$ 


$$
\begin{aligned}
& e_{1 \mathrm{PN}}(\nu)=-\frac{1}{12} \nu-\frac{3}{4} \\
& e_{2 \mathrm{PN}}(\nu)=-\frac{1}{24} \nu^{2}+\frac{19}{8} \nu-\frac{27}{8} \\
& e_{3 \mathrm{PN}}(\nu)=-\frac{35}{5184} \nu^{3}-\frac{155}{96} \nu^{2}+\frac{34445}{576} \nu-\frac{205}{96} \pi^{2} \nu-\frac{675}{64} \\
& e_{4 \mathrm{PN}}^{c}(\nu)=-\frac{3969}{128}+\frac{77}{31104} \nu^{4}+\frac{301}{1728} \nu^{3}+\left(-\frac{498449}{3456}+\frac{3157}{576} \pi^{2}\right) \nu^{2} \\
& +\left(-\frac{123671}{5760}+\frac{1792}{15} \ln (2)+\frac{9037}{1536} \pi^{2}+\frac{896}{15} \gamma\right) \nu \\
& e_{4 \mathrm{PN}}^{\ln }(\nu)=\frac{448}{15} \nu \\
& e_{5 \mathrm{PN}}^{c}(\nu)=-\frac{45927}{512}+\frac{1}{512} \nu^{5}+\frac{55}{512} \nu^{4}+\left(-\frac{1353}{256} \pi^{2}+\frac{69423}{512}\right) \nu^{3} \\
& +\left(-\frac{21337}{1024} \pi^{2}+3 a_{6}^{c 1}-\frac{896}{5} \ln (2)-\frac{448}{5} \gamma+\frac{893429}{2880}\right) \nu^{2} \\
& +\left(-\frac{228916843}{115200}-\frac{9976}{35} \gamma+\frac{729}{7} \ln (3)-\frac{23672}{35} \ln (2)+\frac{126779}{512} \pi^{2}\right) \nu \\
& e_{5 \mathrm{PN}}^{\ln }(\nu)=-\frac{4988}{35} \nu-\frac{656}{5} \nu^{2} \\
& e_{5.5 \mathrm{PN}}(\nu)=\frac{10}{3} \nu\left(\frac{13696}{525} \pi+\nu a_{6.5}^{c 1}\right) \\
& e_{6 \mathrm{PN}}^{c}(\nu)=-\frac{264627}{1024}+\frac{2717}{6718464} \nu^{6}+\frac{5159}{248832} \nu^{5}+\left(\frac{272855}{124416} \pi^{2}-\frac{20543435}{373248}\right) \nu^{4} \\
& +\left(\frac{1232}{27} \gamma+\frac{6634243}{110592} \pi^{2}-\frac{11}{2} a_{6}^{c 1}-\frac{71700787}{51840}+\frac{2464}{27} \ln (2)\right) \nu^{3} \\
& +\left(\frac{113176680983}{14515200}+\frac{18491}{2304} \pi^{4}+\frac{246004}{105} \ln (2)+\frac{112772}{105} \gamma+\frac{11}{2} a_{6}^{c 1}+\frac{2}{3} a_{7}^{\ln 1}\right. \\
& \left.+\frac{11}{3} a_{7}^{c 1}-\frac{86017789}{110592} \pi^{2}-\frac{2673}{14} \ln (3)\right) \nu^{2}+\left(-\frac{389727504721}{43545600}+\frac{74888}{243} \ln (2)\right. \\
& \left.-\frac{7128}{7} \ln (3)-\frac{30809603}{786432} \pi^{4}-\frac{3934568}{8505} \gamma+\frac{9118627045}{5308416} \pi^{2}\right) \nu \\
& e_{6 \mathrm{PN}}^{\ln }(\nu)=-\frac{1967284}{8505} \nu+\frac{24464}{135} \nu^{3}+\frac{39754}{105} \nu^{2}+\frac{11}{3} \nu^{2} a_{7}^{\ln 1} .
\end{aligned}
$$

Note the complexity of this expansion compared with the simplicity of the PN expansion of the EOB $A$ potential, Eqs. (59)- (63). In particular, while $A(u ; \nu)$ is linear in $\nu$ up to the $3 \mathrm{PN}$ level included, and is only quadratic in $\nu$ at the $4 \mathrm{PN}$ level, $E_{B}(x) / \mu$ has a $O\left(\nu^{2}\right)$ contribution at $2 \mathrm{PN}$, a $O\left(\nu^{3}\right)$ one at $3 \mathrm{PN}$, and a $O\left(\nu^{4}\right)$ one at $4 \mathrm{PN}$. This simplicity of the $\nu$ dependence of the EOB radial potential $A(u ; \nu)$ (which, however, contains the same information as the function $\left.E_{B}(x)\right)$ is due to the remarkable cancellations (recalled above) taking place when computing $A(u ; \nu)$ from the PN Hamiltonian. There is, currently, no deep explanation for these cancellations. However, it is tempting to interpret them along the following lines. The GSF (i.e. $\nu$-dependent) part, say $A_{\mathrm{GSF}}(u, \nu)$, of the
$A$ potential $\left[A(u ; \nu) \equiv 1-2 u+A_{\mathrm{GSF}}(u, \nu)\right]$ enters, at lowest order, and when considering the zero angular momentum case, the full Hamiltonian through the contribution $H_{\mathrm{GSF}}=\mu A_{\mathrm{GSF}}(u, \nu)$. Let us consider a certain term in $A_{\mathrm{GSF}}(u, \nu)$ of the form $a_{n+1, p} u^{n+1} \nu^{p}$, i.e. a term at the $n$-PN level, which is proportional to $\nu^{p}$ (with an integer $p \geq 1$ ). If we now insert the expressions for $\mu$, $\nu$, and $u$ in terms of the two masses $m_{1}, m_{2}$, and of the (EOB) radial coordinate $r$, we find that the contribution to $H_{\mathrm{GSF}}=\mu A_{\mathrm{GSF}}(u, \nu)$ corresponding to $a_{n+1, p} u^{n+1} \nu^{p}$ is of the form $\left(m_{1} m_{2}\right)^{p+1}\left(m_{1}+m_{2}\right)^{n-2 p} / r^{n+1}$. When $n$ is an integer, the latter contribution to the Hamiltonian will be a polynomial in the two masses (as one would expect to be the case, at least for an Hamiltonian 
computed in a usual gauge, such as the Arnowitt-DeserMisner one) if and only if the power $p$ of $\nu$ satisfies the inequality $2 p \leq n$. In turn, this inequality says that: (i) at $1 \mathrm{PN}(n=1)$, there cannot exist a $p \geq 1$ satisfying this condition; (ii) at $2 \mathrm{PN}(n=2), p$ can only be equal to 1 ; (ii) at $3 \mathrm{PN}(n=3), p$ can still only be equal to 1 ; while (iv) at $4 \mathrm{PN}(n=4), p$ can only take the values 1 or 2 . All these consequences of the above requirement of polynomiality in the masses (i.e. the inequality $2 p \leq n$ ) exactly coincide with what was found, by explicit computations, at 1, 2, 3 and 4PN (namely, absence of a $1 \mathrm{PN}$ contribution, linearity in $\nu$ at $2 \mathrm{PN}$ and $3 \mathrm{PN}$, and quadratic nonlinearity in $\nu$ at $4 \mathrm{PN})$. This interpretation cannot be considered as a real "explanation" of the cancellations found in $A(u, \nu)$, because the EOB Hamiltonian has not been proven to be directly obtainable by solving Einstein's equations by some kind of usual perturbation theory which keeps the polynomiality in the masses apparent at each stage. However, its phenomenological success in recovering known facts suggests it has some truth. [Note that, by contrast, the function $E_{B}(x ; \nu)$, which is not a Hamiltonian, does not exhibit polynomiality properties in the two masses, even if we generously interpret the variable $x$ as being essentially of the form $G\left(m_{1}+m_{2}\right) / c^{2} r$. For instance, the presence of a term linear in $\nu$ in the coefficient of the $1 \mathrm{PN}$ contribution $e_{1 \mathrm{PN}}(\nu)$ corresponds to a term in $E_{B}\left(G\left(m_{1}+m_{2}\right) / c^{2} r ; \nu\right)$ proportional to $\mu \nu\left(G\left(m_{1}+m_{2}\right) / c^{2} r\right)^{2}$ and therefore proportional to $\left(m_{1} m_{2}\right)^{2} /\left(m_{1}+m_{2}\right)$.] Using our interpretation of the simplicity of $A(u ; \nu)$ as a heuristic basis for discussing higher PN orders, it suggests that: (v) at 5PN $(n=5), p$ can only take the values 1 or 2 (like at $4 \mathrm{PN})$; while (vi) at $6 \mathrm{PN}(n=6), p$ can only take the values 1,2 and 3. [The 5.5PN contribution has to be treated as being offscale, because it is anyway not polynomial in $1 / r$.] In terms of the parametrisation introduced above of the $\nu$ dependence beyond the 1GSF level, the "predictions" (v) and (vi) mean that $a_{6}^{c 1}(\nu)$ would not depend on $\nu$, while $a_{7}^{c 1}(\nu), a_{7}^{\ln 1}(\nu)$ would be at most linear in $\nu$. In addition, the argument given in the next subsection suggests that $a_{6.5}(\nu)$ does not depend on $\nu$, i.e. that $a_{6.5}^{c 1}(\nu)$ vanishes.

If these suggested restricted $\nu$ dependences of the EOB-potential expansion coefficients $a_{n}(\nu)$ turn out to be confirmed, many of the contributions to the bindingenergy function $E_{B}(x ; \nu)$ displayed above (those containing high powers of $\nu$ ) will be already correctly predicted by our (1GSF-based) results. For instance, the terms in $\nu^{4}, \nu^{5}$ and $\nu^{6}$ in the $\nu$-exact $\mathrm{PN}$ expansion of $e_{6 \mathrm{PN}}^{c}(\nu)$ might already be the ones explicitly appearing in the equations written above. A partial confirmation of this prediction concerns the terms in $E_{B}(x ; \nu)$ that are related to the terms in the center-of-mass Hamiltonian which are of maximal order in $\nu$ at some $\mathrm{PN}$ approximation. It has been recently emphasized in Ref. [44 that, at the $n$th PN order, the $\nu^{n}$ terms in the $\mu$-rescaled center-of-mass Hamiltonian carry at most one power of $G$ (see, e.g., [45]), and suffice to determine the terms proportional to $\nu^{n}$ in $e_{n \mathrm{PN}}(\nu)$. Starting from the closed-form, firstpost-Minkowskian (i.e. $O(G)$-accurate) Hamiltonian of Ref. [46], we explicitly computed (for $n=1,2,3,4,5,6$ ) the $\nu^{n}$ terms in the expansion coefficients $e_{n \mathrm{PN}}(\nu)$ of the function $E_{B}(x ; \nu)$ and verified that they agree with the corresponding terms explicitly appearing in Eqs. (74) above: e.g. $+\frac{1}{512} \nu^{5}$ at $5 \mathrm{PN}$, and $+\frac{2717}{6718464} \nu^{6}$ at $6 \mathrm{PN}$. [The corresponding $5 \mathrm{PN}$ result in [44], namely $+\frac{3121}{32} \nu^{5}$, appears to be incorrect.] This agreement confirms the usefulness of the EOB formalism which automatically encodes this information in the very simple ( $\nu$-independent) $1 \mathrm{PN}$-accurate EOB radial potential $A_{1 \mathrm{PN}}(u)=1-2 u$. We have more generally proven that this agreement holds to all $\mathrm{PN}$ orders. [This shows that, at the $n$-PN level, the power of $\nu$ in $\nu a_{n+1}(\nu)=\sum_{p} a_{n+1, p} \nu^{p}$, satisfies the inequality $p \leq n-1$, which is generally weaker than the inequality $2 p \leq n$ conjectured above.] Let us also note a generalization of the above property to the subleading order in the $\nu$ expansion: we have verified that the terms proportional to $\nu^{(n-1)}$ in $e_{n \mathrm{PN}}(\nu)$ are entirely encoded (for $n=1,2,3,4)$ in the simple ( $\nu$-linear) $2 \mathrm{PN}$-accurate EOB radial potential $A_{2 \mathrm{PN}}(u ; \nu)=1-2 u+2 \nu u^{3}$. This property will also hold at $5 \mathrm{PN}$ and $6 \mathrm{PN}$ if the currently unknown $5 \mathrm{PN}$ and $6 \mathrm{PN}$ coefficients have a limited-degree polynomial dependence on $\nu$ (e.g. $a_{6}^{c 1}(\nu)$ would have to involve $\nu^{2}$ to modify the $\nu^{4}$ term in the $5 \mathrm{PN}$ energy contribution $\left.e_{5 \mathrm{PN}}(\nu)\right)$.

\section{B. Possible effect of the second-order tail integral in the inner metric and value of the $5.5 \mathrm{PN}$ coefficient $a_{6.5}(\nu)$}

The presence of a conservative contribution in the inner metric (and thereby in the redshift variable) at the odd $\mathrm{PN}$ order $1 / c^{11}$ (namely the $5.5 \mathrm{PN}$ contributions $h_{6.5}$, $a_{6.5}$, or $e_{5.5 \mathrm{PN}}$ in the results above) is a priori surprising because conservative effects are traditionally associated with even powers of $1 / c$, while odd powers of $1 / c$ are usually associated with time-odd radiation-reaction effects. Let us show here, by a reasoning directly based on $\mathrm{PN}$ theory, why the $5.5 \mathrm{PN}$ order is the first order at which a conservative effect can involve an odd power of $1 / c$, and why the corresponding term must precisely be of the form we found in our RWZ calculations above.

The first point is that (as found in [23]), starting at the $4 \mathrm{PN}$ level, there are hereditary effects (given by an integral over the past behavior of the source) in the inner metric of the system that enter radiation-reaction. Moreover, Ref. 23 found that the hereditary effects in the inner metric are time asymmetric without being time anti-symmetric. As a consequence they have both a dissipative aspect (hereditary modification of the lowest-order $\left(O\left(1 / c^{5}\right)\right)$, Burke-Thorne [47, 48], 2.5PN radiation reaction) and a conservative one (related to the $4 \mathrm{PN}, O\left(1 / c^{8}\right)$, logarithmic contribution to $\left.A(u, \nu)[22]\right)$. [The PN bookkeeping here is that the lowest-order tailrelated hereditary integral discussed in 23] has a prefac- 
tor $G M / c^{3}$, which combines with the leading-order $(2.5$ $\mathrm{PN}$, i.e. $1 / c^{5}$ ) radiation-reaction to go to the $4 \mathrm{PN}$ level $1 / c^{8}$.] In addition, Ref. 24] found that the hereditary integral entering the inner metric obtained in [23] was related to a similar hereditary integral ("radiative tail") entering the wave-zone metric by energy balance between the system and radiative losses at infinity (see Sec. IIID of 24]). As the latter energy-balance link between tail effects in gravitational radiation at infinity and hereditary integrals in the inner metric must clearly be generally satisfied, one expects that higher-order tail effects in gravitational radiation will have corresponding hereditary counterparts in the inner metric, which will modify radiation reaction by integrals having both dissipative aspects and conservative ones. [Like the leading-order hereditary effects, the higher-order hereditary effects being time asymmetric without being time antisymmetric can generate both radiation-reaction-like and conservative effects (thereby violating the usual PN lore).]

The next-to-leading-order quadrupolar tail effect arises from a mass-mass-quadrupole interaction [25], and will carry a prefactor $\left(G M / c^{3}\right)^{2}$. In that case, energy balance indicates that the second-order wave-zone tail in the radiative quadrupole moment (as seen at infinity) of the system [25] will similarly correspond to a timeasymmetric modification of the leading-order (2.5 PN) radiation reaction. The $\mathrm{PN}$ bookkeeping now tells us that the $\left(G M / c^{3}\right)^{2}$ prefactor combines with $O\left(1 / c^{5}\right)$ to go to $O\left(1 / c^{11}\right)$, i.e. the $5.5 \mathrm{PN}$ level. [Dimensional analysis shows that other tail interactions, e.g. of the massquadrupole-quadrupole type, start contributing only at higher PN levels: 6.5PN and higher.] Let us now show in detail how this time asymmetric (but not time anti-symmetric) modification of radiation reaction gives rise precisely to the $5.5 \mathrm{PN}$ conservative contributions to $h_{k k}(u)$ and $A(u ; \nu)$ we found above.

Denoting the radiative quadrupole moment (evaluated at the retarded time $U$ ) as $M_{i j}^{\mathrm{rad}}(U)$, and using a superscript $(n)$ to denote a $n$th time derivative, the result of [25] (which was expressed in terms of $U_{i j} \equiv M_{i j}^{\mathrm{rad}(2)}$ ) can be written as

$$
\begin{aligned}
& M_{i j}^{\mathrm{rad}}(U)=M_{i j}(U) \\
& +2 \frac{G \mathcal{M}}{c^{3}} \int_{0}^{+\infty} d \tau M_{i j}^{(2)}(U-\tau) \ln \left(\frac{c \tau}{2 r_{0}^{\prime}}\right) \\
& +2\left(\frac{G \mathcal{M}}{c^{3}}\right)^{2} \int_{0}^{+\infty} d \tau M_{i j}^{(3)}(U-\tau) \times \\
& \times\left[\ln ^{2}\left(\frac{c \tau}{2 r_{0}^{\prime}}\right)+B \ln \left(\frac{c \tau}{2 r_{0}^{\prime}}\right)+C\right]+\ldots \\
& + \text { semi - hereditary terms }
\end{aligned}
$$

Here, $\mathcal{M}$ denotes the total mass-energy of the system, $M_{i j}$ denotes the algorithmic quadrupole moment of the Blanchet-Damour multipolar-post-Minkowskian formalism [49], $r_{0}^{\prime}=r_{0} \exp (-11 / 12)$ is a length scale differing from the basic length scale $r_{0}$ entering the latter formalism by having absorbed the well-known additional term $\frac{11}{12}$ entering the first-order radiative tail [24], and the final ellipsis indicate some semi-hereditary (memory-type) terms that will not contribute to the near-zone effects discussed below (which involve the 5 th derivative of $M_{i j}^{\mathrm{rad}}$ ). After the absorption of the latter $\frac{11}{12}$ in $r_{0}^{\prime}$, the value of the coefficient $B$ multiplying $\ln \left(\frac{c \tau}{2 r_{0}^{\prime}}\right)$ in the second-order hereditary term is

$$
B=-\frac{107}{105}
$$

The explicit expression given in Eq. (40) above for the crucial 5.5 PN coefficient $A_{11}^{\text {up }(H G, l=2)}$ entering the nearzone expansion of the "up" solution (and thereby the near-zone expansion of the Green's function, and of the metric) shows that it contains (notably in a term proportional to $X_{1}^{2} X_{2}^{7 / 2} \propto \Omega^{7} M^{2}$ which corresponds to what we discuss here) the first power of the logarithm of $2 \omega r \eta$, but not its second power. This shows that the part of the second-order tail integral Eq. (75) involving a squared logarithm does not generate a corresponding near-zone modification of radiation reaction. This is consistent with the finding of Blanchet [25] that, when computing the energy radiated at infinity by circular orbits, the effect linked to the squared logarithm in the tail integral (75) cancels with a term in the square of the first-order tail integral. Let us note in passing that another way of understanding why the term $\ln ^{2}\left(\frac{c \tau}{2 r_{0}^{\prime}}\right)$ in the tail integral does not contribute to the energy loss is via the scale dependence of the algorithmic moment $M_{i j}[23,25,50,51]$ : as the value of $M_{i j}$ depends on the arbitrary length scale $r_{0}^{\prime}$, but the physics does not depend on this choice, one can choose a scale $r_{0}^{\prime}$ so as to simplify the result of the tail integrals. Choosing $r_{0}^{\prime}=r_{*}^{\prime}:=c /\left(4 \Omega \mathrm{e}^{\gamma}\right)$ makes the first-order tail integral [52] $\int_{0}^{+\infty} d \tau \exp (-2 i \Omega \tau) \ln \left(\frac{c \tau}{2 r_{0}^{\prime}}\right)$ purely real, and makes the second-order $\ln ^{2}$ tail integral $\int_{0}^{+\infty} d \tau \exp (-2 i \Omega \tau) \ln ^{2}\left(\frac{c \tau}{2 r_{0}^{\prime}}\right)$ purely imaginary. One then sees, without doing any calculation (taking into account the factors $(2 i \Omega)^{n}$ implied by the $n$th derivatives acting on $M_{i j}$ ) that this implies that the energy loss must of the form $\frac{1}{5} M_{i j}^{(3)}\left[r_{*}^{\prime}\right] M_{i j}^{(3)}\left[r_{*}^{\prime}\right]\left(1+a \pi \mathcal{M} \Omega+\left(b \pi^{2}+c\right)(\mathcal{M} \Omega)^{2}\right)$, with some rational coefficients $a, b, c$. The algorithmic quadrupole moment $M_{i j}\left[r_{*}^{\prime}\right]$ used here corresponds to the special scale $r_{*}^{\prime}$ defined above. Using the $3 \mathrm{PN}$ logarithmic running of $M_{i j}$ with the scale $r_{0}^{\prime}$ discussed in 23, 25, 50, 51], namely

$$
M_{i j}\left[r_{*}^{\prime}\right]=M_{i j}\left[r_{0}^{\prime}\right]+2 B \ln \left(\frac{r_{*}^{\prime}}{r_{0}^{\prime}}\right)\left(\frac{G \mathcal{M}}{c^{3}}\right)^{2} M_{i j}^{(2)}\left[r_{0}^{\prime}\right]
$$

we can then obtain the energy loss for an arbitrary scale. We thereby find that it will contain, at $3 \mathrm{PN}$ order, only the first power of a logarithm (with a factor $\propto B \mathcal{M}^{2}$ ). This reasoning shows also why the coefficient of the logarithmic running is equal (as we have just indicated) to $2 B$, where we recall that $B$ was defined as being the coefficient of the first power of the logarithm in the secondorder tail in Eq. (75). Indeed, when directly performing 
(as in 25]) the calculation of the energy loss with an algorithmic moment corresponding to an arbitrary scale $r_{0}^{\prime}$, the cancellation of the squared logarithms leaves a term linear in $\ln \left(\frac{r_{0}^{\prime}}{r_{*}^{\prime}}\right)=\ln \left(4 \Omega \mathrm{e}^{\gamma} r_{0}^{\prime} / c\right)$ with a coefficient equal to $16 B$. This result is consistent with the reasoning above because the factor 16 is the product of: the factor 2 apparent in Eq. (77), a factor $2^{2}$ from the second derivative $\left(M_{i j}^{(2)}=-(2 \Omega)^{2} M_{i j}\right)$, and a factor 2 from squaring $M_{i j}^{(3)}$.

The above reasoning (together with the results at first order in the tail [23, 24]) suggests that the hereditary effect of the second-order tail in the inner metric consists in modifying the part of the inner metric connected to quadrupolar radiation reaction, i.e.

$$
g_{00}^{\text {radreac }}=-\frac{2 G}{5 c^{7}} x^{i} x^{j} M_{i j}^{(5)}
$$

by adding to $M_{i j}$ (taken at a scale corresponding to the size of the source) twice the contribution with coefficient $B$ in Eq. (75) (see Section III D of [24] for a discussion of this factor 2). Note that the energy balance reasoning gives a handle only on hereditary terms, and not on instantaneous terms, such as the term associated with the coefficient $C$ in Eq. (75). Anyway, the latter term is easily seen to be purely reactive (indeed it modifies $M_{i j}^{(5)}$ into $\left.\sim C \mathcal{M}^{2} M_{i j}^{(7)}\right)$. By contrast, the contribution involving the integral of $B \ln \left(\frac{c \tau}{2 r_{0}^{\prime}}\right)$, i.e.

$g_{00}^{\text {tail }^{2}}=$

$-\frac{2 G}{5 c^{7}} x^{i} x^{j}\left(4 B\left(\frac{G \mathcal{M}}{c^{3}}\right)^{2} \int_{0}^{+\infty} d \tau M_{i j}^{(8)}(U-\tau) \ln \left(\frac{c \tau}{2 r_{0}^{\prime}}\right)\right)$

contains conservative effects. Evaluating the tail integral along a circular orbit, using (see [52])

$$
\int_{0}^{+\infty} d y \ln y \mathrm{e}^{-\sigma y}=-\frac{1}{\sigma}(\ln \sigma+\gamma)
$$

and computing the contribution of the metric (799) to the Hamiltonian, then leads to a contribution (involving the logarithmic sum $S_{7,2}^{\text {log }}$ ) to the EOB radial potential of a (comparable-mass) binary system equal to

$$
A^{\mathrm{tail}^{2}}(u, \nu)=-\frac{2^{7}}{5} B \pi \nu u^{13 / 2}=+\frac{13696}{525} \pi \nu u^{13 / 2} .
$$

In the limit $\nu \rightarrow 0$ this reproduces our result Eq. (67b) above, but we get the new information that $a_{6.5}(\nu)$ is actually independent of $\nu$. [This independence essentially follows from the facts that $\mathcal{M}=M+O\left(1 / c^{2}\right)$ and that the Newtonian quadrupole moment is proportional to $\mu r_{12}^{2}$, where the interbody distance $r_{12}$ is also related to $M$ and $\Omega$ by Kepler's law.]

Though the reasoning that led us to this result seems physically justified, let us emphasize that, at this stage, it is only heuristic. One will need a matching argument between the inner metric and the wave-zone one (along the lines of Refs. [19, 23]) to fully justify the final result. However, we find that the above reasoning clarifies the appearance of a conservative contribution at the 5.5 PN order, and strongly suggests that our ( $\nu$-linear) $1 \mathrm{GSF}$ result Eq. 67b remains valid for any value of $\nu$. [Ref. [26], which appeared on the archives soon after our work, has confirmed the result of our analysis by a detailed multipolar-post-Minkowskian treatment of the gravitational field outside the source.]

\section{CONCLUSIONS}

Let us summarize our method and results:

We have studied several gauge-invariant functions characterizing the energetics of binary systems in the limit $m_{1} \ll m_{2}$. At linear order in the (symmetric) mass ratio $\nu=m_{1} m_{2} /\left(m_{1}+m_{2}\right)^{2}$, these functions are linked by simple relations. On the one hand, Detweiler's redshift function $h_{k k}^{R}(u):=h_{\mu \nu}^{R} k^{\mu} k^{\nu}$ is related to its other avatars through Eqs. (13) and (14). On the other hand, it is related to the $O(\nu)$ piece $a(u)$ in the main EOB radial potential $A(u ; \nu)$, Eq. (65), through Eq. (44). Then, from $A(u ; \nu)$ one can compute the function relating the binding energy of a binary to the orbital frequency by Eqs. (68), (69), (70), (71), (72). [At linear order in $\nu$, the link between $h_{k k}$ and the binding energy was first derived in [9]; however, we prefer to use the EOB link between $A(u ; \nu)$ and $E_{B}(x)$ because it is valid to all orders in $\nu$.]

We used gravitational self force techniques to compute $h_{k k}^{R}(u)$ up to the $6 \mathrm{PN}$ accuracy, included. The details of our method (which we already used in our previously reported $4 \mathrm{PN}$-level computation) are spelled out in Sec. II. The final, $6 \mathrm{PN}$ accurate, result for $h_{k k}^{R}(u)$ is given in Eq. (57). The corresponding $6 \mathrm{PN}$-accurate result for $a(u)$ is given in Eqs. 65), 67a , 67b), 67c . Combining this information with the full $\nu$-dependent $4 \mathrm{PN}$ knowledge of $A(u ; \nu)$, and with a conjecture interpreting the remarkable cancellations of the $\nu$ dependence of $A(u ; \nu)$ up to $4 \mathrm{PN}$ (see the end of the previous Section), we obtained Eqs. (74) for the $6 \mathrm{PN}$-accurate energy function $E_{B}(u, \nu)$ (including part of the higher powers of $\nu$, notably the $\nu^{n}$ terms in $\left.e_{n \mathrm{PN}}(\nu)\right)$.

An interesting result of our work is the finding of a 5.5PN contribution (i.e. of order $1 / c^{11}$ ) to the energetics of binary systems. As we discussed in the text, though this term, which is conservative, seems to conflict with the usual PN lore that conservative (time-even) effects arise at even powers of $1 / c$, and that odd powers of $1 / c$ are associated with time-odd radiation-reaction effects, it is simply interpreted in terms of the result of Blanchet and Damour 23. . We have sketched the generalization of the result of [23] to second order in the tail factor 25] and found that it indeed explains the value we found (at the $1 \mathrm{GSF}$ approximation) for the $5.5 \mathrm{PN}$ contribution to $A(u ; \nu)$, and suggests it is valid for any value of $\nu$. The important conceptual point here is that the hereditary 
tail effects are time dissymetric without being time antisymmetric. They can thereby generate terms that are either radiation-reaction-like or conservative (thereby violating the usual PN lore).

Ref. 22] has shown that the 1GSF shift of the orbital frequency of the last stable (circular) orbit was given (in terms of the dimensionless frequency parameter $x$ ) by $x_{\mathrm{LSO}}=\frac{1}{6}\left(1+c_{x} \nu\right)$ (corresponding to $\left(m_{1}+m_{2}\right) \Omega_{\mathrm{LSO}}=$ $6^{-3 / 2}\left(1+c_{\Omega} \nu\right)$ with $\left.c_{\Omega}=\frac{3}{2} c_{x}\right)$, where $c_{x}=\tilde{a}(1 / 6)+$ $c_{x}^{E}$, with a universal part $c_{x}^{E}=\frac{2}{3}\left(1-\sqrt{\frac{8}{9}}\right)$ and a part depending on the derivatives of the EOB $a$ potential:

$$
\tilde{a}\left(\frac{1}{6}\right)=a\left(\frac{1}{6}\right)+\frac{1}{6} a^{\prime}\left(\frac{1}{6}\right)+\frac{1}{18} a^{\prime \prime}\left(\frac{1}{6}\right) .
$$

The numerical value of $\tilde{a}\left(\frac{1}{6}\right)$ was estimated by means of accurate GSF simulations in [28], and found to be

$$
\tilde{a}^{\operatorname{num}}\left(\frac{1}{6}\right)=0.795883004(15)
$$

(corresponding to $c_{\Omega}^{\text {num }}=1.251015464(23)$ ). By contrast, if we use our $6 \mathrm{PN}$-accurate result (66) for $a(u)$, we find a value

$$
\tilde{a}^{6 P N}\left(\frac{1}{6}\right)=0.8495304315
$$

(corresponding to $\left.c_{\Omega}^{6 P N}=1.331486606\right)$.

As we see, the $6 \mathrm{PN}$ expansion of $\tilde{a}(u)$ yields an estimate of $\tilde{a}\left(\frac{1}{6}\right)$ which differs from $\tilde{a}^{\text {num }}\left(\frac{1}{6}\right)$ by $6.7 \%$. This is another example of the poor convergence of PN approximants in the (semi-) strong field regime (here, $u=G M / c^{2} r_{0}=\frac{1}{6}$ ). This poor convergence is linked with the presence of a (formal) singularity in the function $a(u)$ (and its redshift cousins, such as $h_{k k}$ ) at the light ring $u=\frac{1}{3}$ 28]. Indeed, this singularity implies that the PN expansion coefficients $a_{n}$ grow with $n$ like $\sim 3^{n}$. As a consequence the $(n-1)$-PN contribution to $\tilde{a}\left(\frac{1}{6}\right)$ coming from $a_{n}$ is expected to decrease, as $n$ increases, only like $\left(2 n^{2}-n+1\right) a_{n} / 6^{n} \sim 2 n^{2} / 2^{n}$ (see Eq. (4.35) in [22]). For instance, the $6 \mathrm{PN}$ contribution linked to the non-logarithmic coefficient $a_{7} \simeq 738.28$ is equal to 0.24263 , which is far from being small, and actually represents $30 \%$ of the final value of $\tilde{a}\left(\frac{1}{6}\right)$.

Let us finally comment on the comparison of our exact analytical higher-PN results to previous numerical estimates. In terms of the coefficients of the PN expansion of $a(u)$, the numerical values of the new coefficients we computed (including the previously reported $4 \mathrm{PN}$ one [1]) are

$$
\begin{aligned}
a_{5} & \approx+23.503389242603436240 \\
a_{6} & \approx-134.07179509567553064 \\
a_{6.5} & \approx+81.956672349649158466 \\
a_{7} & \approx+738.2786376500962441 \\
a_{7}^{\ln } & =-\frac{2522}{405} \approx-6.2271604938271604938 .
\end{aligned}
$$

[We do not list here the logarithmic coefficients $a_{5}^{\text {ln }}$ and $a_{6}^{\ln }$ that were analytically known before, and that our work has confirmed.]

Previous accurate numerical investigations of Detweiler's redshift function led to corresponding numerical estimates for these coefficients equal to [7, 19].

$$
\begin{aligned}
a_{5}^{\text {num }} & \approx+23.50190(5) \\
a_{6}^{\text {num }} & \approx-137.72(1) \\
a_{7}^{\text {num }} & \approx+118(2) \\
a_{7}^{\text {ln,num }} & =-255.0(5) .
\end{aligned}
$$

The comparison between the two sets of values shows an increasing level of discrepancy as the PN order increases. As already noted in 1], even at the 4PN-level, the analytical value of the coefficient $a_{5}$ differs by 30 standard deviations from its previous numerical estimate. The discrepancy becomes larger and larger for the other coefficients, to the extent that they seem to be completely unrelated at the $6 \mathrm{PN}$ level. However, the reason for these discrepancies is clear. Ref. [19] had not allowed for a 5.5PN contribution, i.e. had effectively set $a_{6.5}$ to zero. As a consequence their fit had to absorb the missing $5.5 \mathrm{PN}$ term by different values of the $6 \mathrm{PN}$ coefficients $a_{7}^{\text {num }}, a_{7}^{\text {ln,num }}$. The missing "signal" associated with $a_{6.5} u^{6.5}$ had also an effect on the determination of the lower PN coefficients $a_{5}^{\text {num }}$, and $a_{6}^{\text {num }}$ (with, as expected, a stronger effect on the closest PN level). To check the idea that the discrepancies between our analytical estimates and the previous analytical ones are entirely due to the non inclusion of a $5.5 \mathrm{PN}$ term in the expansion, we have directly compared our $6 \mathrm{PN}$-accurate analytical prediction for the quantity $h_{u u}^{R}$ (which was the quantity fitted in [19]) to the best corresponding fitting polynomial obtained in the latter reference (with coefficients denoted $a_{j}$ and $b_{j}$ there). We found that the difference $h_{u u}^{\text {analyt }}-h_{u \mu}^{\text {numeri }}$ stayed within the error level for $h_{u u}$ estimated in [19].

The results reported above were obtained during the writing of our short report [1]. The preprint of Shah et al. 20] (which appeared on the archives just before the submittal of our work) have very recently provided new, ultra-high-accuracy numerical results on Detweiler's redshift variable $u_{(1 G S F)}^{t}$. They give also numerical evidence, and an analytical argument, for a $5.5 \mathrm{PN}$ contribution to $u_{(1 G S F)}^{t}$. To compare our results with theirs, one must re-express our analytical $5 \mathrm{PN}, 5.5 \mathrm{PN}$ and $6 \mathrm{PN}$ results in terms of the variable $u_{(1 G S F)}^{t}$. For this variable (which is related via Eq. (14) to $h_{k k}$ ) our analytical results for the $6 \mathrm{PN}$-accurate expansion read 


$$
\begin{aligned}
& u_{(1 G S F)}^{t}=-u-2 u^{2}-5 u^{3}+\left(-\frac{121}{3}+\frac{41}{32} \pi^{2}\right) u^{4} \\
& +\left(\frac{677}{512} \pi^{2}-\frac{128}{5} \gamma-\frac{256}{5} \ln (2)-\frac{1157}{15}-\frac{64}{5} \ln u\right) u^{5} \\
& +\left(\frac{1606877}{3150}-\frac{60343}{768} \pi^{2}+\frac{1912}{105} \gamma+\frac{7544}{105} \ln 2+\frac{956}{105} \ln u-\frac{243}{7} \ln 3\right) u^{6} \\
& -\frac{13696}{525} \pi u^{13 / 2} \\
& +\left(\frac{17083661}{4050}+\frac{102512}{567} \gamma+\frac{51256}{567} \ln u+\frac{372784}{2835} \ln 2-\frac{1246056911}{1769472} \pi^{2}+\frac{1215}{7} \ln 3+\frac{2800873}{262144} \pi^{4}\right) u^{7}
\end{aligned}
$$

In the notation of [19], i.e.

$$
u_{(1 G S F)}^{t}=\sum_{j}\left(\alpha_{j}+\beta_{j} \ln u\right) u^{j+1}
$$

the higher-order PN coefficients, including the nonlogarithmic 4PN one derived in [1], read (with the index $j$ now denoting the $\mathrm{PN}$ level)

$$
\begin{aligned}
\alpha_{4} & =\frac{677}{512} \pi^{2}-\frac{128}{5} \gamma-\frac{256}{5} \ln (2)-\frac{1157}{15} \\
& =-114.34895136757260295 \ldots \\
\alpha_{5} & =\frac{1606877}{3150}-\frac{60343}{768} \pi^{2}+\frac{1912}{105} \gamma+\frac{7544}{105} \ln 2-\frac{243}{7} \ln 3 \\
& =-243.176814464674307587 \ldots \\
\beta_{5} & =+\frac{956}{105} \\
& =9.1047619047619047619 \ldots \\
\alpha_{5.5} & =-\frac{13696}{525} \pi \\
& =-81.9566723496491584647 \ldots \\
\alpha_{6} & =\frac{17083661}{4050}+\frac{102512}{567} \gamma+\frac{372784}{2835} \ln 2-\frac{1246056911}{1769472} \pi^{2}+\frac{1215}{7} \ln 3+\frac{2800873}{262144} \pi^{4} \\
& =-1305.00138107870965574 \ldots \\
\beta_{6} & =+\frac{51256}{567} \\
& =90.39858906525573192239 \ldots
\end{aligned}
$$

These numerical values of our analytical results agree with the values determined numerically (to a very high accuracy) in [20]. Our analytical result for $\alpha_{5.5}$ agrees also with their independent result (as well as that of [26]). We note also that our fully analytical derivation of the $6 \mathrm{PN}$ logarithmic coefficient $\beta_{6}$ agrees with its numerical-analytical determination in [20].
Appendix A: Relation $\nu$ vs $l$ at higher orders in $\epsilon$

In Eq. (35) we have cited (from 12 14]) the first term in the expansion of $\nu$ in powers of $\epsilon^{2}$. Let us exhibit here (for the case $s=-2$ of interest for us) explicit analytical expressions for the next two terms, of order $\epsilon^{4}$, and $\epsilon^{6}$ :

$$
\nu=l+\nu_{2}(l) \epsilon^{2}+\nu_{4}(l) \epsilon^{4}+\nu_{6}(l) \epsilon^{6}+O\left(\epsilon^{8}\right)
$$

We find 
$\nu_{2}(l)=-\frac{1}{2} \frac{\left(15 l^{4}+30 l^{3}+28 l^{2}+13 l+24\right)}{l(3+2 l)(1+2 l)(-1+2 l)(l+1)}$

$\nu_{4}(l)=-\frac{1}{8} \frac{\bar{\nu}_{4}}{(5+2 l)(-1+l)(1+2 l)^{3}(2+l)(-3+2 l)(3+2 l)^{3}(-1+2 l)^{3}(l+1)^{3} l^{3}}$

$\nu_{6}(l)=-\frac{1}{16} \frac{\bar{\nu}_{6}}{(-5+2 l)(1+2 l)^{5}(7+2 l)(5+2 l)^{2}(-1+l)^{2}(2+l)^{2}(-3+2 l)^{2}(3+2 l)^{5}(-1+2 l)^{5}(l+1)^{5} l^{5}}+c_{6}\left(\delta_{2}^{l}-\delta_{2}^{-l-1}\right)$.

where

$\bar{\nu}_{4}=18480 l^{16}+147840 l^{15}+456120 l^{14}+605640 l^{13}+8295 l^{12}-1096830 l^{11}-1678310 l^{10}-1520455 l^{9}$

$-1355518 l^{8}-1397512 l^{7}-1217380 l^{6}-733273 l^{5}+675625 l^{4}+1855326 l^{3}+850608 l^{2}-102816 l-51840$

$\bar{\nu}_{6}=-12975033600 l-2216596603241 l^{16}-2756712215600 l^{15}+69152667840 l^{2}+276057468576 l^{3}-595527565392 l^{4}$

$-3036767042412 l^{5}-2535956468604 l^{6}+2930850757357 l^{7}-3984546031837 l^{13}-3214558957703 l^{12}+6222948337606 l^{8}$

$+3813852229717 l^{9}-3680916388051 l^{14}-1750882207589 l^{11}+345064441861 l^{10}+938280719684 l^{19}-1215830743322 l^{18}$

$+9306705408 l^{28}+104552448 l^{30}+1568286720 l^{29}-509720642816 l^{23}+527411908408 l^{22}-525016804928 l^{24}$

$-187730326784 l^{25}+2143419358696 l^{21}-2130563709104 l^{17}+2569889990822 l^{20}-1170321152 l^{26}$

$+24173140992 l^{27}-3919104000$.

and where the coefficient $c_{6}$ of the last, additional term in $\nu_{6}(l)$ involving the Kronecker symbols $\delta_{2}^{l}$ and $\delta_{2}^{-l-1}$ (which only contribute when $l=2$ or $l=-3$ ) is equal to

$$
c_{6}=\frac{14}{1605}
$$

As pointed out to us by Ryuichi Fujita (private communication) the presence (say in the case $l=2$ ) of such an additional term is linked to the irregular behavior (as functions of $\epsilon$ ) of the coefficients $\alpha_{n}^{\nu}$ and $\gamma_{n}^{\nu}$ for the negative values $n=-l-1$ and $n=-l$ of the index $n$. [Note that the term $O\left(\epsilon^{6}\right)$ corresponds to the $9 \mathrm{PN}$ order, and did not enter the derivation of the results reported here.] The functions $\nu_{2 k}(l)(k=1,2,3)$ are antisymmetric around $l=-\frac{1}{2}$, i.e. they satisfy

$$
\nu_{2 k}(l)+\nu_{2 k}(-l-1)=0 .
$$

In the cases $l=2,3,4$ used in the present work their values are listed below.

\begin{tabular}{|l||c|c|c|}
\hline$l$ & $\nu_{2}(l)$ & $\nu_{4}(l)$ & $\nu_{6}(l)$ \\
\hline 2 & $-\frac{107}{210}$ & $-\frac{1695233}{9261000}$ & $-\frac{76720109901233}{480698687700000}$ \\
\hline 3 & $-\frac{13}{42}$ & $-\frac{10921}{271656}$ & $-\frac{95353832269}{7709149047600}$ \\
\hline 4 & $-\frac{1571}{6930}$ & $-\frac{68482418369}{4326563241000}$ & $-\frac{6966001672062143707}{2701175970905111700000}$ \\
\hline
\end{tabular}

Acknowledgments. T.D. thanks Francis Brown and Pierre Deligne for informative discussions about the origin of the transcendentality structure of the higher-PN coefficients $a_{n}(0)$, Steven Detweiler for clarifying email exchanges about the regularization of $h_{k k}$, and Luc Blanchet for discussions about higher-order tail effects involving several multipoles. We thank Ryuichi Fujita for informative email exchanges about the relation $\nu$ vs $l$ at higher orders in $\epsilon$, and Stefano Foffa for attracting our attention towards the $\nu^{n}$ terms in $e_{n \mathrm{PN}}$. We are grateful to ICRANet for partial support. D.B. thanks IHES for hospitality during crucial stages of development of this 
project.

[1] D. Bini and T. Damour, "Analytical determination of the two-body gravitational interaction potential at the 4th post-Newtonian approximation," Phys. Rev. D 87, 121501(R) (2013) arXiv:1305.4884 [gr-qc].

[2] A. Buonanno and T. Damour, "Effective one-body approach to general relativistic two-body dynamics," Phys. Rev. D 59, 084006 (1999) gr-qc/9811091.

[3] A. Buonanno and T. Damour, "Transition from inspiral to plunge in binary black hole coalescences," Phys. Rev. D 62, 064015 (2000) gr-qc/0001013.

[4] T. Damour, P. Jaranowski and G. Schaefer, "On the determination of the last stable orbit for circular general relativistic binaries at the third postNewtonian approximation," Phys. Rev. D 62, 084011 (2000) gr-qc/0005034.

[5] T. Damour, "Coalescence of two spinning black holes: an effective one-body approach," Phys. Rev. D 64, 124013 (2001) gr-qc/0103018.

[6] P. Jaranowski and G. Schäfer, "Dimensional regularization of local singularities in the 4th post-Newtonian twopoint-mass Hamiltonian," Phys. Rev. D 87, 081503(R) (2013) arXiv:1303.3225 [gr-qc].

[7] E. Barausse, A. Buonanno and A. Le Tiec, "The complete non-spinning effective-one-body metric at linear order in the mass ratio," Phys. Rev. D 85, 064010 (2012) arXiv:1111.5610 [gr-qc]].

[8] A. Le Tiec, L. Blanchet and B. F. Whiting, "The First Law of Binary Black Hole Mechanics in General Relativity and Post-Newtonian Theory," Phys. Rev. D 85 (2012) 064039 arXiv:1111.5378 [gr-qc]].

[9] A. Le Tiec, E. Barausse and A. Buonanno, "Gravitational Self-Force Correction to the Binding Energy of Compact Binary Systems," Phys. Rev. Lett. 108, 131103 (2012) arXiv:1111.5609 [gr-qc]].

[10] S. L. Detweiler, "A Consequence of the gravitational selfforce for circular orbits of the Schwarzschild geometry," Phys. Rev. D 77, 124026 (2008) arXiv:0804.3529 [gr-qc]].

[11] L. Barack and A. Ori, "Mode sum regularization approach for the selfforce in black hole space-time," Phys. Rev. D 61, 061502 (2000) gr-qc/9912010.

[12] S. Mano, H. Suzuki and E. Takasugi, "Analytic solutions of the Teukolsky equation and their low frequency expansions," Prog. Theor. Phys. 95, 1079 (1996) gr-qc/9603020.

[13] S. Mano, H. Suzuki and E. Takasugi, "Analytic solutions of the Regge-Wheeler equation and the postMinkowskian expansion," Prog. Theor. Phys. 96, 549 (1996) gr-qc/9605057.

[14] S. Mano and E. Takasugi, "Analytic solutions of the Teukolsky equation and their properties," Prog. Theor. Phys. 97, 213 (1997) gr-qc/9611014.

[15] N. Sago, H. Nakano and M. Sasaki, "Gauge problem in the gravitational selfforce. 1. Harmonic gauge approach in the Schwarzschild background," Phys. Rev. D 67, 104017 (2003) gr-qc/0208060.

[16] H. Nakano, N. Sago and M. Sasaki, "Gauge problem in the gravitational selfforce. 2. First postNewtonian force under Regge-Wheeler gauge," Phys. Rev. D 68, 124003 (2003) gr-qc/0308027.
[17] W. Hikida, S. Jhingan, H. Nakano, N. Sago, M. Sasaki and T. Tanaka, "A New analytical method for selfforce regularization. II. Testing the efficiency for circular orbits," Prog. Theor. Phys. 113, 283 (2005) gr-qc/0410115.

[18] W. Hikida, H. Nakano and M. Sasaki, "Self-force regularization in the Schwarzschild spacetime," Class. Quant. Grav. 22, S753 (2005) gr-qc/0411150.

[19] L. Blanchet, S. L. Detweiler, A. Le Tiec and B. F. Whiting, "High-Order Post-Newtonian Fit of the Gravitational Self-Force for Circular Orbits in the Schwarzschild Geometry," Phys. Rev. D 81, 084033 (2010) arXiv:1002.0726 [gr-qc]].

[20] A. G. Shah, J. L. Friedman and B. F. Whiting, "Finding high-order analytic post-Newtonian parameters from a high-precision numerical self-force calculation," arXiv:1312.1952 [gr-qc].

[21] T. Damour, 2010, unpublished; cited in L. Barack, T. Damour and N. Sago, "Precession effect of the gravitational self-force in a Schwarzschild spacetime and the effective one-body formalism," Phys. Rev. D 82, 084036 (2010) arXiv:1008.0935 [gr-qc]], which quoted and used some combinations of the logarithmic contributions to $a(u)$ and $\bar{d}(u)$.

[22] T. Damour, "Gravitational Self Force in a Schwarzschild Background and the Effective One Body Formalism," Phys. Rev. D 81, 024017 (2010) arXiv:0910.5533 [grqc]].

[23] L. Blanchet and T. Damour, "Tail Transported Temporal Correlations In The Dynamics Of A Gravitating System," Phys. Rev. D 37, 1410 (1988).

[24] L. Blanchet and T. Damour, "Hereditary effects in gravitational radiation," Phys. Rev. D 46, 4304 (1992).

[25] L. Blanchet, "Gravitational wave tails of tails," Class. Quant. Grav. 15, 113 (1998) [Erratum-ibid. 22, 3381 (2005)] gr-qc/9710038.

[26] L. Blanchet, G. Faye and B. F. Whiting, "Half-integral conservative post-Newtonian approximations in the redshift factor of black hole binaries," arXiv:1312.2975 [grqc].

[27] L. Blanchet, S. L. Detweiler, A. Le Tiec and B. F. Whiting, "Post-Newtonian and Numerical Calculations of the Gravitational Self-Force for Circular Orbits in the Schwarzschild Geometry," Phys. Rev. D 81, 064004 (2010) arXiv:0910.0207] [gr-qc]].

[28] S. Akcay, L. Barack, T. Damour and N. Sago, "Gravitational self-force and the effective-one-body formalism between the innermost stable circular orbit and the light ring," Phys. Rev. D 86, 104041 (2012) arXiv:1209.0964 [gr-qc]].

[29] S. L. Detweiler, E. Messaritaki and B. F. Whiting, "Selfforce of a scalar field for circular orbits about a Schwarzschild black hole," Phys. Rev. D 67, 104016 (2003) gr-qc/0205079].

[30] N. Sago, L. Barack and S. L. Detweiler, "Two approaches for the gravitational self force in black hole spacetime: Comparison of numerical results," Phys. Rev. D 78, 124024 (2008) arXiv:0810.2530 [gr-qc]]. 
[31] F. J. Zerilli, "Gravitational field of a particle falling in a schwarzschild geometry analyzed in tensor harmonics," Phys. Rev. D 2, 2141 (1970).

[32] L. Barack and C. O. Lousto, "Perturbations of Schwarzschild black holes in the Lorenz gauge: Formulation and numerical implementation," Phys. Rev. D 72, 104026 (2005) gr-qc/0510019.

[33] S. L. Detweiler and E. Poisson, "Low multipole contributions to the gravitational selfforce," Phys. Rev. D 69, 084019 (2004) gr-qc/0312010.

[34] S. Chandrasekhar, Proc. R. Soc. London A 343, 289 (1975).

[35] L. Blanchet and T. Damour, "Multipolar radiation reaction in general relativity," Phys. Lett. A 104, 82 (1984).

[36] E. Poisson and M. Sasaki, "Gravitational radiation from a particle in circular orbit around a black hole. 5: Black hole absorption and tail corrections," Phys. Rev. D 51, 5753 (1995) gr-qc/9412027.

[37] M. Sasaki and H. Tagoshi, "Analytic black hole perturbation approach to gravitational radiation," Living Rev. Rel. 6, 6 (2003) gr-qc/0306120.

[38] J. L. Anderson, L. S. Kegeles, R. G. Madonna and R. E. Kates, "Divergent Integrals Of Postnewtonian Gravity: Nonanalytic Terms In The Near Zone Expansion Of A Gravitationally Radiating System Found By Matching," Phys. Rev. D 25, 2038 (1982).

[39] T. Damour and G. Schaefer, "Higher Order Relativistic Periastron Advances And Binary Pulsars," Nuovo Cim. B 101, 127 (1988).

[40] P. Jaranowski and G. Schaefer, "Third postNewtonian higher order ADM Hamilton dynamics for two-body point mass systems," Phys. Rev. D 57, 7274 (1998) [Erratum-ibid. D 63, 029902 (2001)] gr-qc/9712075].

[41] T. Damour, P. Jaranowski and G. Schaefer, "Dimensional regularization of the gravitational interaction of point masses," Phys. Lett. B 513, 147 (2001) gr-qc/0105038.
[42] P. Jaranowski and G. Schafer, "Towards the 4th postNewtonian Hamiltonian for two-point-mass systems," Phys. Rev. D 86, 061503 (2012) arXiv:1207.5448 [grqc]].

[43] T. Damour and G. Esposito-Farese, "Testing gravity to second postNewtonian order: A Field theory approach," Phys. Rev. D 53, 5541 (1996) gr-qc/9506063.

[44] S. Foffa, "Gravitating binaries at 5PN in the postMinkowskian approximation," Phys. Rev. D 89, 024019 (2014) arXiv:1309.3956 [gr-qc]].

[45] T. Damour, P. Jaranowski and G. Schäfer, "Non-localin-time action for the fourth post-Newtonian conservative dynamics of two-body systems," arXiv:1401.4548 [gr-qc].

[46] T. Ledvinka, G. Schäfer and J. Bicak, "Relativistic Closed-Form Hamiltonian for Many-Body Gravitating Systems in the Post-Minkowskian Approximation," Phys. Rev. Lett. 100, 251101 (2008) arXiv:0807.0214 [gr-qc]].

[47] W. L. Burke, "Gravitational Radiation Damping Of Slowly Moving Systems Calculated Using Matched Asymptotic Expansions," J. Math. Phys. 12, 401 (1971).

[48] K. S. Thorne, "Nonradial Pulsation of GeneralRelativistic Stellar Models.IV. The Weakfield Limit," Astrophys. J. 158, 997 (1969).

[49] L. Blanchet and T. Damour, "Radiative gravitational fields in general relativity I. general structure of the field outside the source," Phil. Trans. Roy. Soc. Lond. A 320, 379 (1986).

[50] L. Blanchet, B. R. Iyer and B. Joguet, "Gravitational waves from inspiralling compact binaries: Energy flux to third postNewtonian order," Phys. Rev. D 65, 064005 (2002) [Erratum-ibid. D 71, 129903 (2005)] gr-qc/0105098.

[51] W. D. Goldberger and A. Ross, "Gravitational radiative corrections from effective field theory," Phys. Rev. D 81, 124015 (2010) arXiv:0912.4254 [gr-qc]].

[52] L. Blanchet and G. Schaefer, "Gravitational wave tails and binary star systems," Class. Quant. Grav. 10, 2699 (1993). 